\title{
Preconditioning Therapy with Lentiviral Vector-Programmed Dendritic Cells Accelerates the Homeostatic Expansion of Antigen-Reactive Human T Cells in NOD.Rag1 ${ }^{-/-} . I L-2 r \gamma C^{-/-}$mice
}

\author{
Gustavo Salguero,, Bala Sai Sundarasetty, Sylvia Borchers,, Dirk Wedekind, ${ }^{2}$ Britta Eiz-Vesper, ${ }^{3}$ \\ Sarvari Velaga, Adan C. Jirmo, ${ }^{4}$ Georg Behrens, ${ }^{4}$ Gregor Warnecke, ${ }^{5}$ Ann-Kathrin Knöfel, \\ Rainer Blasczyk, Eva Mischak-Weissinger, Arnold Ganser, and Renata Stripecke ${ }^{1}$
}

\begin{abstract}
Dendritic cell (DC)-based immunization is a potent strategy to direct prompt and durable immune responses against viral reactivations after transplantations. Here, we show that overnight lentiviral vector (LV) gene transfer into human monocytes co-expressing granulocyte-macrophage colony stimulating factor and interleukin (IL)-4 induced self-differentiated DCs (SMART-DCs) with stable DC immunophenotype over weeks in culture and secreted several inflammatory cytokines. SMART-DCs injected subcutaneously in immunodeficient NOD.Rag1 $1^{-/-}$.IL2 $r \gamma^{-/-}$(NRG) mice 1 day after LV transduction were stable for a month in vivo. "Conventional" DCs (cDCs) and SMART-DCs were compared with regard to their potency to accelerate the expansion, biodistribution, and antigenic stimulation of autologous human T cells. Peripheral blood cells obtained from human cytomegalovirus (hCMV)-reactive donors and full-length hCMV pp65 antigenic protein or peptides were used. DCs loaded with pp65 were administered subcutaneously into NRG mice as a preconditioning treatment a week prior to intravenous infusion with $\mathrm{T}$ cells. Optical imaging analyses demonstrated that in mice preconditioned with SMART-DC-pp65, T cells were directly recruited to the immunization site and subsequently spread to the spleen and other organs. A dramatic expansion of both human $\mathrm{CD}^{+}$and $\mathrm{CD} 4^{+} \mathrm{T}$ cells could be observed within a few days after infusion, and this was associated with consistent measurable CD8 ${ }^{+}$effector memory T-cell responses against different pp65 epitopes. Thus, this mouse model demonstrates the proof-ofprinciple for SMART-DCs to accelerate expansion of human lymphocytes, resulting in poly-functional and antigen-specific immune responses against hCMV-pp65.
\end{abstract}

\section{Introduction}

A DULT PATIENTS RECEIVING lympho-depleting regimens such as chemotherapy and hematopoietic stem cell transplantation present severe deficiencies in the $\mathrm{CD}^{+}$and $\mathrm{CD}^{+}$T-cell populations (Small et al., 1999). During this lymphopenic period, subjects are at high risk of life-threatening opportunistic infections and leukemia relapse. Clinical studies have demonstrated that adoptive therapy with small numbers of donor leukocytes can be associated with partial restoration of $\mathrm{CD}^{+}$and $\mathrm{CD}^{+}$T-cell numbers, antigenspecific T-cell responses, and resolution of viral-associated diseases (Small et al., 1999). The use of donor lymphocyte infusion (DLI, also known as donor lymphocyte transfer) has become a standard procedure for the treatment of high-risk leukemia and is capable of producing a strong graft-versusleukemia effect (Kolb, 2008). Several factors such as the host, conditioning regimen, and type of allograft influence the T-cell reconstitution and ultimately the efficacy of DLI. In young hosts, T-cell immune reconstitution following lymphodepletion can occur through active thymopoiesis, but for older adults, it is most likely to occur through homeostatic proliferation of peripherally expanded clones (Williams et al., 2007). Homeostatic peripheral expansion results in rapid, significant expansion of the T-cell pool, and is dependent upon both support of homeostatic cytokines and antigen-driven

\footnotetext{
${ }^{1}$ Department of Hematology, Hemostasis, Oncology and Stem Cell Transplantation, ${ }^{2}$ Institute of Animal Laboratory Science, ${ }^{3}$ Institute for Transfusion Medicine, ${ }^{4}$ Clinic for Immunology and Rheumatology, ${ }^{5}$ Department of Cardiac, Thoracic Transplantation and Vascular Surgery, Hannover Medical School, D-30625 Hannover, Germany.
} 
responses in the period following lymphopenia (Williams et al., 2007).

Dendritic cells (DCs) are potent antigen-presenting cells (APCs) that play a pivotal role in antigen presentation and co-stimulation of naïve and memory $\mathrm{CD}^{+}$and $\mathrm{CD}^{+} \mathrm{T}$ cells. There is a limited amount of detailed information regarding the recovery in numbers and co-stimulatory activity of DCs after allogeneic or autologous transplantation, but it is estimated that DC recovery may take several weeks and the DC numbers rarely reach the levels observed in healthy controls (Horvath et al., 2009). DCs constitute an infrequent cell population of the hematopoietic system (less than $1 \%$ of the circulating peripheral blood mononuclear cells) and dysfunction or fluctuations in their numbers have been implicated in the risk of chronic infections (Anthony et al., 2004). Therefore, accelerating the repopulation of DCs and stabilizing their function in the lymphopenic host might enhance homeostatic peripheral expansion of $\mathrm{T}$ cells and improve the DLI effects.

Several clinical trials have been conducted worldwide using DCs produced ex vivo as cellular adjuvants to boost immunity against cancer (Figdor et al., 2004). One of the major issues with the standardization of DC vaccines is their variable quality and viability, which ultimately affects their performance in T-cell stimulation in vivo (Figdor et al., 2004). Possibly due to the low in vivo viability and engraftment of DCs maintained ex vivo for several days, it is estimated that less than $5 \%$ of intradermally administered mature DCs reach the draining lymph nodes (De Vries et al., 2003).

As a novel technique for DC generation, we have devised a 1-day gene reprogramming strategy that induces monocytes to autonomously self-differentiate into stable DCs in vitro (Koya et al., 2004). This can be successfully achieved by the use of self-inactivating third-generation lentiviral vectors (LVs), a robust gene transfer platform that has expanding applicability in the immunotherapy and immunization fields (Pincha et al., 2010). Ex vivo transduction of monocytes with designed LVs induced DC differentiation through co-expression of granulocyte-macrophage colonystimulating factor (GM-CSF), interleukin (IL)-4, and melanoma-associated antigens, demonstrating a practical and superior alternative to conventional DCs (cDCs) for inducing $\mathrm{CD}^{+}$cytotoxic T lymphocytes (CTLs) to prevent and treat melanoma in immunocompetent mice (Koya et al., 2007). These self-differentiated myeloid-derived antigenpresenting cells reactive against tumors (SMART-DC̄s) were extensively tested as cell vaccines in syngeneic mouse models for melanoma immunotherapy (Koya et al., 2007; Pincha et al., 2011) and demonstrated no pathology (malignancies or autoimmunity) concerns.

In this study, we explored human SMART-DCs expressing a model viral antigen, the cytomegalovirus (CMV) pp65 protein. The aim of this study was to establish the proof-ofconcept of preconditioning the host with SMART-DCs prior to T-cell infusion, in order to accelerate the engraftment, biodistribution, and stimulation of antigen-specific immune responses. As an in vivo model system, we employed the immunodeficient NOD.Rag1 ${ }^{-/-} . \mathrm{IL} 2 \mathrm{r} \gamma^{-/-}$(NRG) radioresistant nonobese diabetic (NOD) mouse containing mutations in the recombination activating gene-1 (Rag1 $\left.1^{\text {null }}\right)$ and IL-2 receptor common gamma chain (IL2r $\gamma^{\text {null }}$ ) (Pearson et al., 2008). NRG mice have a long life span, despite severe adaptive and innate immune deficiencies. T, B, and natural killer (NK) cells are absent, allowing a permissive environment for reconstitution of a functional human immune system. It has been demonstrated that human peripheral blood mononuclear cells (PBMCs) injected intravenously at high doses $\left(20 \times 10^{6}\right)$ could engraft in NRG adult mice, and 4 weeks later $\mathrm{CD}^{+}{ }^{+}$and $\mathrm{CD} 8^{+}$T-cell subsets could be found in spleen and peripheral blood (Pearson et al., 2008). Here, we compared the effects of cDCs and SMART-DCs in NRG mice on the engraftment, biodistribution, and function of $\mathrm{T}$ cells. We monitored the dynamic of human T-cell biodistribution and homing patterns in NRG mice by a combination of optical imaging analyses, histology, flow cytometry, and antigen-specific immunological assays.

\section{Materials and Methods}

\section{Cells lines}

Human embryonic kidney 293T cells (obtained from ATCC) were cultured in Dulbecco's modified Eagle's medium with $10 \%$ fetal bovine serum and penicillin $(100 \mathrm{U} / \mathrm{ml})$ and streptomycin $(100 \mathrm{mg} / \mathrm{ml})$.

\section{LV construction and virus production}

The self-inactivating lentiviral backbone vector and the monocistronic vectors expressing firefly luciferase (RRLcPPT-CMV-fLUC) and green fluorescent protein (RRL-cPPTCMV-GFP) were previously described (Kimura et al., 2007). Construction of the bicistronic lentiviral vector LV-G24 containing the cDNAs of the human GM-CSF and of the human IL-4 interspaced with a P2A element (RRL-cPPTCMV-hGMCSF-P2A-hIL4) was constructed by overlapping polymerase chain reaction (PCR) essentially as described previously (Szymczak and Vignali, 2005). Primers used to generate the interspacing P2A element between hGM-CSF and hIL-4 genes were P2A/IL-4 forward 5'-GGATCCG GAGCCACGAACTTCTCTCTGTTAAAGCAAGCAGGAGA CGTGGAAGAAAACCCCGGTCCTGCCACCATGGGTCTC ACCTCCCAAC-3' and P2A/GM-CSF reverse 5'-GTCTCCT GCTTGCTTTAACAGAGAGAAGTTCGTGGCTCCGGATC CCTCCTGGACTGGCTCCCAGCA-3'. For generation of LV expressing the CMV pp65 protein (LV-pp65), cDNA was PCR amplified from a bacmid (Borst et al., 2007) kindly provided by Prof. M. Messerele (Institute of Virology, Hannover Medical School) with the primers Xba-UL83-L forward 5'-TATTTCTAGAGCCACCATGGAGTCGCGCG GTCGG-3' and Bam-UL83-L reverse 5'-TAATGGATCCC CTTCAACCTCGGTGCTTTTTGGG-3'. The PCR product was digested with the restriction enzymes XbaI and BamHI and introduced into the site of pRRL-sin-cPPT-hCMV-MCS vector. The structural integrity of all constructs was reconfirmed by restriction digestion and sequencing analysis of the promoters and transgenes. Large-scale production of lentiviral constructs was performed by transient cotransfection of 293T cells exactly as described by Stripecke (2009). LVs were concentrated by ultracentrifugation, and lentiviral titer was further determined by assessing viral p24 antigen concentration by enzyme-linked immunoabsorbent assay (ELISA) (Cell Biolabs, Inc.). One microgram p24 equivalent per milliliter corresponds to approximately $(1-5) \times 10^{7}$ infective viral particles $/ \mathrm{ml}$. 
Transduction of 293T cells and monocytes and generation of human SMART-DCs

$293 \mathrm{~T}$ cells were transduced with $0.5 \mu \mathrm{g} / \mathrm{ml}$ p24 equivalent of LVs in the presence of $5 \mu \mathrm{g} / \mathrm{ml}$ protamine sulfate (American Pharmaceutical Partners, Inc.). PBMCs were obtained from HLA-A02*01-positive healthy adult volunteers, and all studies were performed in accordance with protocols approved by the Hannover Medical School Ethics Review Board. For the generation of SMART-DCs, CD14 ${ }^{+}$cells were isolated using CD14 isolation beads (Miltenyi Biotec) and cultured in $\mathrm{X}$-Vivo 15 medium (Lonza) in the presence of recombinant human GM-CSF and IL-4 (50 ng/ml each; Cellgenix) for $8 \mathrm{hr}$ before transduction. For generation of SMART-DCs, $5 \times 10^{6}$ $\mathrm{CD}_{14}{ }^{+}$monocytes were transduced in six-well plates in the presence of $2.5 \mu \mathrm{g}$ p24 equivalent/ml of LV-G24 plus $5 \mu \mathrm{g} / \mathrm{ml}$ protamine sulfate. Sixteen hours post transduction, cells were washed twice with phosphate-buffered saline (PBS) and further cultured in X-Vivo 15 medium or immediately resuspended in PBS and injected into mice. cDCs were generated from $\mathrm{CD} 14^{+}$cells and supplemented with GM-CSF and IL-4 every 3 days. For maturation, cells were treated from day 5 to 7 with IL-1 $\beta 5 \mathrm{ng} / \mathrm{ml}$; IL-6 $10 \mathrm{ng} / \mathrm{ml}$; tumor necrosis factor (TNF)- $\alpha 200 \mathrm{U} / \mathrm{ml}$ (Immunotools) and $\mathrm{PGE}_{2} 1 \mu \mathrm{g} / \mathrm{ml}$ (Sigma). For luciferase marking, monocytes were preincubated with GM-CSF/IL-4, transduced with LV-fLUC alone (Mono/fLUC) or in co-transduction with LV-G24 (SMART-DC/fLUC), washed, and administered subcutaneously into mice. For specific immune responses, SMART-DCs were generated by co-transduction with LV-G24 and LV-pp65 (2.5 $\mu \mathrm{g}$ p24 equivalent $/ \mathrm{ml}$ each). cDCs expressing the pp65 protein were generated by transduction of monocytes with LV-pp65 and further cultured for 7 days in the presence of GM-CSF and IL-4 supplemented every 3 days. cDCs pulsed with pp65 were loaded on day 6 with an overlapping peptide pool covering the whole sequence of the pp65 protein (Miltenyi Biotec) and $24 \mathrm{hr}$ later washed and used for in vitro T-cell stimulation or injected directly into mice.

\section{Analyses of human cytokine expression}

Accumulation of secreted human GM-CSF and IL-4 after LV transduction was evaluated in supernantants obtained from 293T cells, preconditioned monocytes, and SMARTDCs by ELISA. Supernatants were assessed in ELISA microplates specific for human GM-CSF and human IL-4 (R\&D Systems Inc.) according to the manufacturer's instructions. Fourteen-plex Luminex bead kit (Invitrogen) was used to detect cytokines up-regulated in SMART-DCs.

\section{Analyses of pp65 transgene expression}

CMV-pp65 transgene expression was evaluated in 293T cells by fluorescent activated cell sorter (FACS) analysis and immunohistochemistry. 293T cells transduced with LV-pp65 were collected after $72 \mathrm{hr}$ and fixed using the BD cytofix/ cytoperm solution (Becton Dickinson) followed by incubation with BD perm/wash solution (Becton Dickinson), as indicated by the manufacturer's instructions. Cells were further incubated with a fluorescein isothiocyanate (FITC)conjugated mouse monoclonal antibody against CMV-pp65 (Pierce Biotechnology) in a dilution of 1:250, washed, and analyzed by flow cytometry. For immunohistochemistry analysis of pp65 expression, $293 \mathrm{~T}$ cells $\left(2 \times 10^{5}\right)$ were cytospun and further stained with a mouse monoclonal antibody against pp65 (Biotest) followed by alkaline phosphatase detection (mouse Dako REAL ${ }^{\mathrm{TM}}$ Detection System) according to the manufacturer's instructions.

\section{Transduction of human T cells}

Autologous human $\mathrm{T}$ cells recovered from the CD14 ${ }^{+}$ magnetic selection were activated with human anti-CD2/ CD3/CD28-conjugated magnetic beads (Myltenyi Biotec) in a bead-to-cell ratio of 1:2 and cultured in X-Vivo medium in the presence of $200 \mathrm{ng} / \mathrm{ml}$ of human IL-2, $5 \mathrm{ng} / \mathrm{ml}$ of human IL-7, and $5 \mathrm{ng} / \mathrm{ml}$ of IL-15. Cells were culture for $48 \mathrm{hr}$ at a density of $5 \times 10^{6}$ cells per well in a humidified incubator at $37^{\circ} \mathrm{C}$ and $5 \% \mathrm{CO}_{2}$. For lentivirus transduction, cells were spinfected at $200 \times g$ and $32^{\circ} \mathrm{C}$ for $120 \mathrm{~min}$ in the presence of $2.5 \mu \mathrm{g}$ of p24 equivalent/ml of LV-fLUC, LV-rLUC (for in vivo and in vitro luminescence assays, respectively), and LV-GFP (for transduction efficiency determination) and incubated for an additional $16 \mathrm{hr}$. Transduction efficiency was determined by flow cytometry analyses of GFP-positive cells.

\section{Mouse experiments}

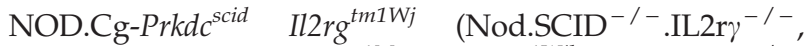
NSG) and NOD.Cg-Rag1 $1^{\text {tm1Mom }}$ Il2rg ${ }^{\text {tm1Wjl }}$ (Nod.Rag1 ${ }^{-/-}$. IL2 $\mathrm{r}^{-1-}$, NRG) mice were bred and maintained under pathogen-free conditions in an IVC system (BioZone) with controlled temperature of $22 \pm 2{ }^{\circ} \mathrm{C}$, relative humidity of approximately $55 \%$, and artificial light from 5:30 to 19:30 hr on a sterilized commercial softwood granulate bedding (Lignocel, Altromin). Health status was monitored according to the Federation of European Laboratory Animal Science Associations recommendations. All procedures involving mice were reviewed and approved by the Lower Saxony State Office for Consumer Protection and Food Safety and followed the guidelines provided by the Animal Facility at the Hannover Medical School. Cells suspensions containing SMART-DCs, cDCs, or monocytes transduced with LV-fLUC $\left(5 \times 10^{5}\right.$ in $100 \mu$ l of PBS) were subcutaneously injected into the mouse hind flank using a 27-gauge needle, and engraftment and viability were evaluated at different time points by in vivo bioluminescence imaging analyses. For in vivo T-cell expansion experiments, mice were primed with cDCs, cDCspp65, SMART-DCs, or SMART-DCs-pp65 $\left(5 \times 10^{5}\right.$ in $100 \mu \mathrm{l}$ of PBS) by subcutaneous injection into the hind flank and 7 days later intravenously infused with fLUC-T cells suspensions $\left(5 \times 10^{6}\right.$ cells in $100 \mu$ l of PBS) into the lateral tail vein. At different time points, engraftment and expansion of fLUC-T cells were evaluated by in vivo bioluminescence imaging analyses. Engraftment of human T cells was further evaluated in peripheral blood by flow cytometry (days 7, 14, and 30 after T-cell infusions). For measurement of immune responses, animals were humanely sacrificed and peripheral blood and spleens were collected for tetramer staining and interferon (IFN)- $\gamma$ enzyme-linked immunospot (ELISPOT) assays, using the schedules as indicated in Results.

\section{In vivo bioluminescence imaging analyses}

Mice were anesthetized with ketamine $(100 \mathrm{mg} / \mathrm{kg}$ intraperitoneally) and xylazine ( $10 \mathrm{mg} / \mathrm{kg}$ intraperitoneally), and 
an aqueous solution of D-luciferin $(150 \mathrm{mg} / \mathrm{kg}$ intraperitoneally) was injected $5 \mathrm{~min}$ before imaging. Animals were placed into a dark chamber of the charge-coupled device camera (IVIS, Xenogen), and gray-scale body surface reference images (digital photograph) were taken under weak illumination. After the light source was switched off, photons emitted from luciferase-expressing cells within the animal body and transmitted through the tissue were quantified over a defined time of up to $5 \mathrm{~min}$ using the software program Living Image (Xenogen) as an overlay on Igor (Wavemetrics). For anatomical localization, a pseudocolor image representing light intensity (blue, least intense; red, most intense) was generated in Living Image and superimposed over the gray-scale reference image. Quantified luminescence consists in averaged photon radiance on the surface of the animal and is expressed as photons $/ \mathrm{sec} / \mathrm{cm}^{2} / \mathrm{sr}$ where $\mathrm{sr}=$ steradian.

\section{Flow cytometry analysis}

The phenotype of conventional or SMART-DC in vitro was analyzed on days 7,14 , and 21 by using a combination of the following antibodies: PerCP-conjugated anti-human CD209, PE-conjugated anti-human CD86, PerCP-conjugated antihuman HLA-DR, and APC-conjugated anti-human CD83 or their respective isotypes (Becton Dickinson). Engraftment of human T cells in mice was evaluated in peripheral blood and spleens using PerCP-conjugated anti-human CD45, FITCconjugated anti-mouse CD45, APC-conjugated anti-human CD3, FITC-conjugated anti-human CD4, and PE-conjugated anti-human CD8 antibodies (Becton Dickinson). For in vitro cultures, cells were collected, washed once with PBS and incubated with mouse IgG $(50 \mu \mathrm{g} / \mathrm{ml})$ on ice for $15 \mathrm{~min}$ followed by incubation with the corresponding monoclonal antibodies for $30 \mathrm{~min}$. Cells were washed, resuspended in cell fix solution (Becton Dickinson), and further analyzed using a FACSCalibur cytometer (Becton Dickinson). For in vivo T-cell expansion experiments, peripheral blood was incubated with antibodies for $15 \mathrm{~min}$ at room temperature followed by erythrocyte lysis buffer incubation (Becton Dickinson). Harvested spleen cells were treated with erythrocyte lysis buffer $(0.83 \%$ ammonium chloride $/ 20 \mathrm{mM}$ Hepes, pH 7.2) for $1 \mathrm{~min}$, washed with PBS and incubated with antibodies for $30 \mathrm{~min}$ on ice. After a washing step, cells were resuspended in cell fix solution and acquired in FACSCalibur flow cytometer (Becton Dickinson). Acquisition and analysis were performed using CellQuest or Dako Cytomation Summit (Dako) software. Additional analysis of lymphocyte subpopulations and regulatory $\mathrm{T}$ cells of in vitro T-cell/DC co-cultures were performed using the following antibodies: FITC-conjugated CD45RA, ECD-conjugated CD3, PCy5-conjugated CD62L, PCy7-conjugated CD8, FITCconjugated CD4, PE-conjugated CD127, ECD-conjugated CD3, and PCy5-conjugated CD25 (Beckman Coulter Inc.). For staining, $1 \times 10^{5}$ stimulated $\mathrm{T}$ cells were incubated for $30 \mathrm{~min}$ at room temperature with antibodies and tetramers, washed twice, and analyzed in a flow cytometer (FC500; Beckman Coulter). The flow-cytometer optical alignment and the fluidic stability were tested prior to analysis of samples using Flow-Check Fluorospheres (Beckman Coulter). At least 5000 CD3/CD8 double positive viable cells were analyzed. Tetramer positive cells are expressed as the percentage of human $\mathrm{CD}^{+} / \mathrm{CD}^{+}$cells.

\section{Histology and immunohistochemistry analysis of human T-cell engraftment}

Spleens from mice engrafted with human $\mathrm{T}$ cells were harvested and embedded in optimal cutting temperature compound (O.C.T., Sakura Finetek) for cryopreservation. Frozen sections $(6 \mu \mathrm{m})$ were fixed by acetone and stained with monoclonal anti-human CD3 (eBioscience), anti-human Pe-Texas Red-conjugated CD8 (eBioscience), and anti-mouse FITC-CD31 mouse (BD Bioscience). Immunofluorescence analyses were performed in a BX61 fluorescence microscope (Olympus) and images created using CellP software.

\section{Stimulation of CMV-reactive T cells in vitro}

We tested the following groups: no APC, cDC, SMARTDCs loaded with pp65 overlapping peptide pool (Miltenyi Biotec). PBMCs from the leukapheresis were thawed, and $\mathrm{CD}^{+}$or $\mathrm{CD}^{+}$cells were enriched by magnetic-activated cell separation (MACS) following the manufacturer's protocol (Miltenyi Biotec). Purity and vitality of enriched cells were checked by FACS. $\mathrm{CD}^{+}$or $\mathrm{CD}^{+}$cells were seeded into a 24 -well plate at $3 \times 10^{6}$ cells/well and autologous DCs were added at a 100:1 ratio (T-cells:DCs). $1 \times 10^{6}$ autologous feeder cells $\left(\mathrm{CD}^{-}\right)$gamma-irradiated with 100 Gy were added to the CD8 T cells. X-Vivo 15 was added to a final volume of $2 \mathrm{ml}$ per well. Cells were kept at $37^{\circ} \mathrm{C}$ for 7 days, and starting from day 3, IL-2 (10 IU / ml) (Novartis Pharma $\mathrm{GmbH}$ ) was added and the medium (with cytokine) was replenished daily. After 7 days, T cells were harvested, washed twice with PBS, and tested for their pp65-specificity by tetramer staining with PE-conjugated pp65-reactive tetramers HLA-A*0201 (NLVPMVATV) and HLA-B*0702 (TPRVTGGGAM), APC-conjugated anti-human CD3, and FITC-conjugated anti-human CD8 (Beckman Coulter Inc.). $\mathrm{T}$-cell subpopulations and regulatory $\mathrm{T}$ cells were assessed by flow cytometry as described above.

\section{Detection of pp65-CTL in mouse peripheral blood}

Quantification of CMV-specific reactive T cells in peripheral blood was assessed by pp65-tetramer staining. Briefly, mouse peripheral blood was collected, stained for $15 \mathrm{~min}$ at room temperature with the tetramers and antibodies, and further incubated with erythrocyte lysis reagent (Beckman Coulter). After a washing step, cells were fixed and analyzed in a flow cytometer.

\section{Functional analyses of pp65-CTLs recovered from mouse spleen}

For functional analysis of pp65-CTLs, splenocytes were harvested, stained with APC-conjugated anti-human CD3, and sorted using a XDP cell sorter (Beckman Coulter). Human $\mathrm{CD}^{+}$cells were seeded at a density of 10,000 cells per well in anti-human IFN- $\gamma$-coated 96-well ELISPOT plate and incubated overnight in the presence of $10 \mu \mathrm{g} / \mathrm{ml}$ of pp65 overlapping peptide pool (Miltenyi). A CEF recall peptide pool corresponding to a mixture of CMV, Epstein-Barr virus, and influenza virus epitopes (PANA Tecs $\mathrm{GmbH}$ ) was used as positive control. The next day, cells were washed and plates were further incubated with biotin-conjugated antihuman IFN- $\gamma$ antibodies followed by alkaline phosphataseconjugated streptavidine. Plates were developed using NBT/ 
BCIP liquid substrate and analyzed in an ELISPOT reader (AELVIS GmbH).

\section{Statistical analysis}

Parametric ( $t$ test) and nonparametric (Kruskal-Wallis) statistical analyses were performed to compare the differences of the viability and biodistribution of human SMART$\mathrm{DC}$ and engraftment/expansion of human $\mathrm{T}$ cells in vivo between the experimental groups (control, cDC, cDC-pp65, SMART-DC, and SMART-DC-pp65). All tests were twosided, and $p<0.05$ was considered significant.

\section{Results}

\section{LVs for expression of GM-CSF, IL-4, and pp65}

We designed a bicistronic lentiviral construct containing 2A elements interspacing the human GM-CSF and IL-4 transgenes (Fig. 1a). The 2A sequence contains a consensus motif (2A, Asp-Val/Ile-Glu- X- Asn- Pro-Gly; 2B, Pro), resulting in cleavage between the $2 \mathrm{~A}$ glycine and $2 \mathrm{~B}$ proline by a ribosomal "skipping" mechanism (Szymczak et al., 2004; Szymczak and Vignali, 2005). We used a monocistronic LV expressing the full-length immune dominant pp65 CMV antigen (encoded by unique long [UL] region 83, which is a tegument protein abundantly synthesized during lytic infection) for the evaluation of CMV-specific immune re- sponses in vivo. We used LVs for the expression of marking genes firefly luciferase (fLUC, for in vivo bio-luminescence image analyses), renilla luciferase (rLUC, for in vitro luciferase assays), and green fluorescent protein (GFP, for flow cytometry) (Fig. 1a). Validation of the bicistronic GM-CSF/ IL-4 LV construct was performed in human embryonic kidney 293T cells. Seven days after transduction, 293T secreted high levels of GM-CSF (average $283.8 \mathrm{ng} / \mathrm{ml}$ ) and IL-4 (average $158.8 \mathrm{ng} / \mathrm{ml}$ ) (Fig. 1b). Expression of CMV-pp65 protein in 293T cells transduced with LV-pp65 was evaluated by intracellular staining followed by flow cytometry (Fig. 1c) and confirmed by immunocytochemistry (Supplementary Fig. S1; Supplementary Data are available online at www.liebertonline.com/hum).

\section{$L V$ transduction of monocytes results in SMART-DCs}

Monocyte-derived SMART-DCs were generated using a protocol previously established in our laboratory (Koya et al., 2004; Stripecke, 2009) (Fig. 2a). CD14 ${ }^{+}$cells isolated from PBMCs obtained from CMV sero-positive healthy volunteers were preconditioned for $8 \mathrm{hr}$ with recombinant human GMCSF and IL-4 in order to enhance transduction efficiency and avoid macrophage overgrowth. Monocytes were cotransduced with LV-G24 and additional viruses (LV-pp65, LV-GFP, or LV-fLUC) for $16 \mathrm{hr}$. Roughly $2.5 \times 10^{7}$ infective viral particles ( $2.5 \mu \mathrm{g}$ p2 4 equivalent) of each $\mathrm{LV}$ were used
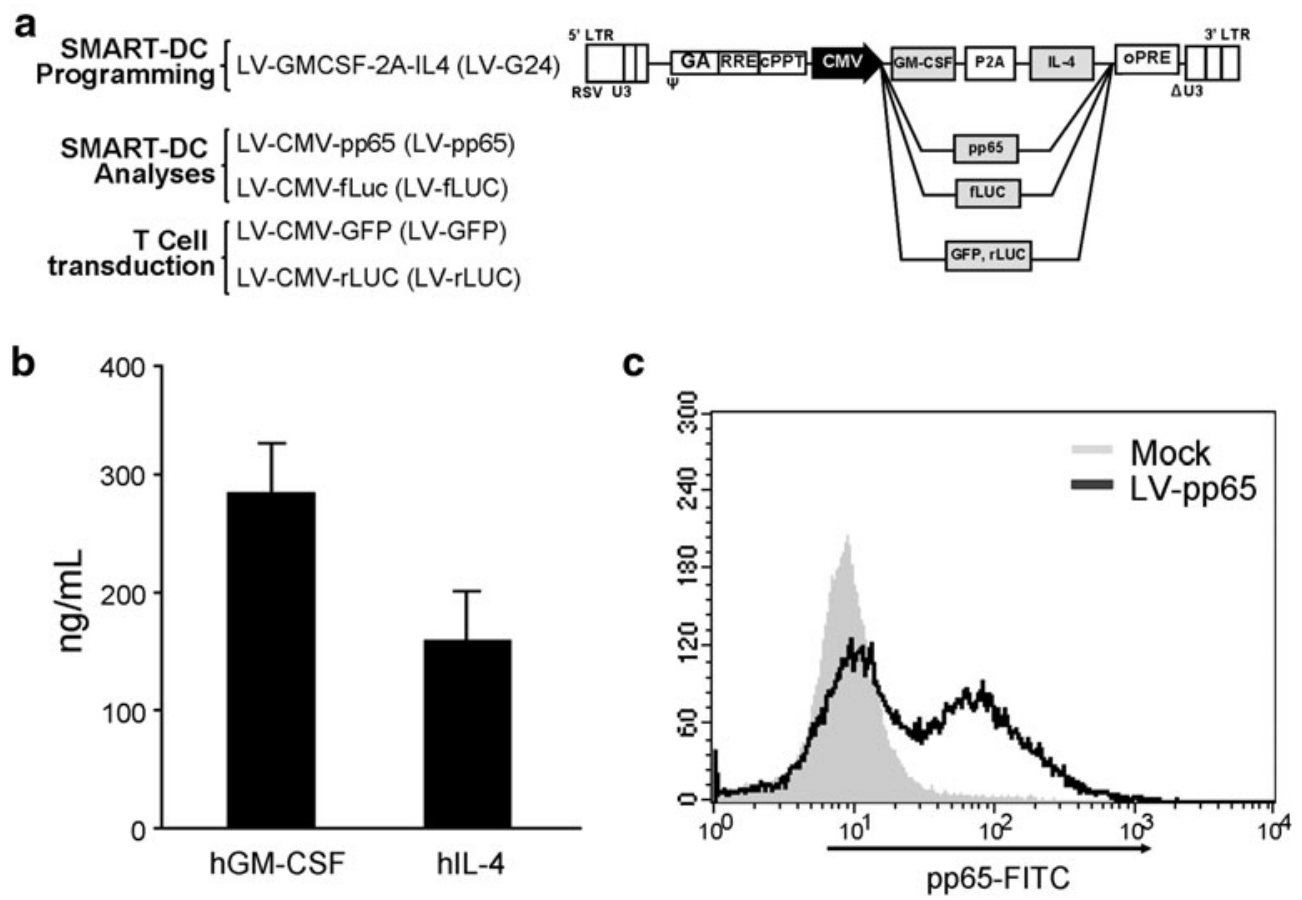

FIG. 1. Lentiviral constructs and validation of transgene expression. (a) Schematic representation of the bicistronic lentiviral construct LV-G24 for simultaneous expression of human granulocyte-macrophage colony-stimulating factor (GM-CSF) and human interleukin (IL)-4 and monocistronic vectors for expression of the cytomegalovirus (CMV) protein pp65, firefly luciferase (fLUC), renilla luciferase (rLUC), and green fluorescent protein (GFP). The chimeric $5^{\prime}$ long terminal repeats (LTR), the packaging signal $(\Psi)$, the truncated and out-of-frame gag gene $(\mathrm{Ga})$, the Rev responsive element (RRE), the central polypurine and termination sequence (cPPT), the CMV early promoter, the non-encoding pre element (opre), and the selfinactivating mutation in the 3' LTR ( $\triangle \mathrm{U} 3$ ) are indicated (not to scale). The bicistronic construct contains the 2A-like sequences from the porcine teschovirus (P2A) upstream of human IL-4. (b) Average levels of human GM-CSF and IL-4 released in supernatants from 293T cells transduced with the bicistronic lentivirus, determined by ELISA in duplicate experiments. (c) Flow cytometry analysis of pp65 expression in 293T cells transduced with LV-pp65. 
a

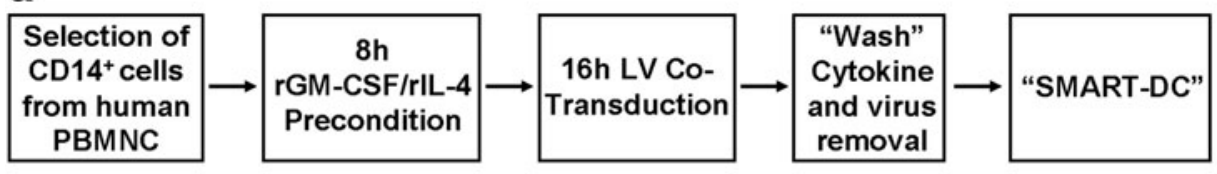

b
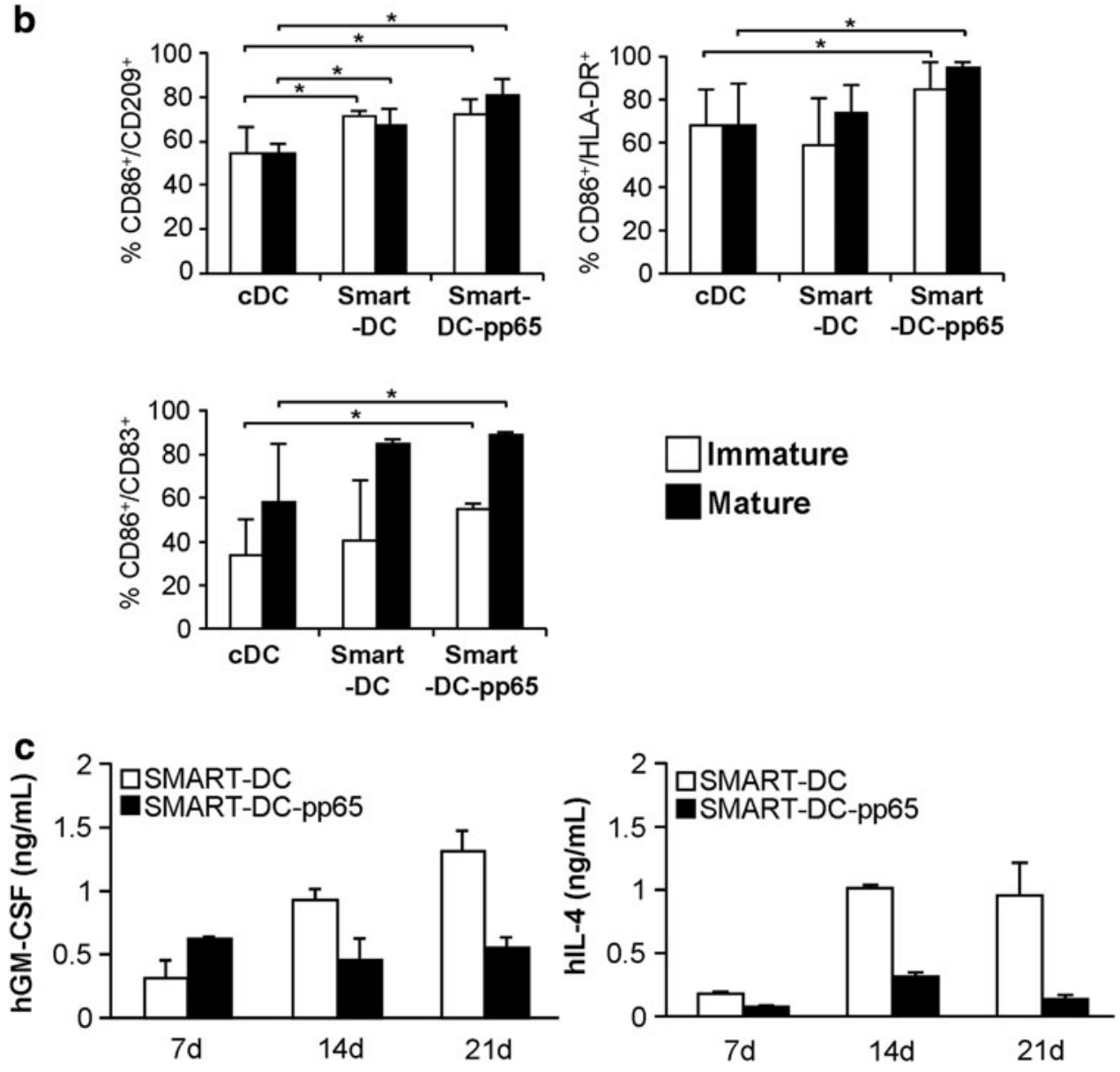

FIG. 2. Generation of human monocyte-derived SMART-DC. (a) Schematic representation of SMARTDC generation. (b) Flow cytometry analyses of cDCs, SMART-DCs, and SMARTDCs expressing the fulllength CMV-pp65 protein (SMART-DCs-pp65) on day 7 of culture. Histogram bars represent the frequency of cells positive for the DC markers. Average and standard deviation were calculated from experiments performed with cells obtained from three different donors. (c) Concentration of human GMCSF (right panel) and IL-4 (left panel) in supernatants harvested from SMART-DC and SMART-DC-pp65 cultures on days 7,14 , and 21. Error bars indicate standard error of mean (SEM). ${ }^{*} p<0.05, n=3$ per group. SMART-DCs, self-differentiated myeloid-derived antigenpresenting cells reactive against tumors; DC dendritic cell; $\mathrm{cD} \overline{\mathrm{C}}$, conventional dendritic cell. PBMNC, peripheral blood mononuclear cell. for transduction of $5 \times 10^{6}$ cells. Using this multiplicity of infection (MOI of 5), approximately $30 \%$ of the monocytes were transduced (as followed by flow cytometry analyses of the co-transduced GFP reporter gene, Supplementary Fig. S2a). After lentiviral transduction, virus and cytokines were washed off and cells were kept in culture for up to 21 days in the absence of exogenously added cytokines. cDCs were generated in parallel by culturing $\mathrm{CD} 14^{+}$cells supplemented every 3 days with fresh human recombinant GM-CSF/IL-4.

Seven days after lentiviral transduction, SMART-DC cultures displayed a typical DC morphology (Supplementary Fig. S2b) and homogeneous DC immunophenotype (70\%$80 \%$ purity) as revealed by the expression of relevant immunologic markers CD209 (DC-SIGN), HLA-DR (MHC-II), and CD86 (a co-stimulatory molecule) (Fig. 2b). Analyses performed at different time-points (7, 14, and 21 days of culture) demonstrated an overall a higher purity of SMARTDCs compared with cDCs (Supplementary Fig. S2b). Higher frequency of mature DCs (approximately $50 \%$ of the cells, defined by $\mathrm{CD} 209^{+} / \mathrm{CD} 3^{+}$co-expression) were observed in the SMART-DCs, compared with cDCs (Fig. 2b). Upon treatment with a cytokine maturation mix, SMART-DCs showed a higher frequency of mature cells $(80 \%-90 \%)$ than cDCs (average $50 \%$ ). Confirming our previous observations (Koya et al., 2004, 2007), SMART-DCs started losing viability from day 21 onwards (data not shown).

Accumulated levels of GM-CSF and IL-4 in supernatants of SMART-DC and SMART-DC-pp65 cultures were measured weekly by ELISA for a period of 3 weeks (Fig. 2c). High levels of cytokines in cell culture supernatants were detected throughout the culture period, peaking on day 21 (SMART-DCs, $1.3 \mathrm{ng} / \mathrm{ml}$ for GM-CSF and $0.9 \mathrm{ng} / \mathrm{ml}$ for IL-4; SMART-DCs-pp65, $0.5 \mathrm{ng} / \mathrm{ml}$ for GM-CSF and $0.14 \mathrm{ng} / \mathrm{ml}$ for IL-4; Fig. 2c). Preconditioned monocytes controls maintained in culture from 7 to 21 days released trace amounts of GM-CSF (average $0.07 \mathrm{ng} / \mathrm{ml}$ ) and IL-4 (average 0.012 ng/ $\mathrm{ml}$; Fig S2c). Multiplex bead array analysis of accumulated cytokines secreted by cDCs and SMART-DCs after 7 days of culture $(n=3)$ demonstrated significant higher levels of several pro-inflammatory cytokines (IL- $1 \beta$, TNF- $\alpha$, IL-6, IL-8) and one chemokine (MCP-1) in supernatants obtained from SMART-DCs than cDCs (Table 1). In addition, SMART-DCs produced detectable levels of IL-2, IL-5, IL-7, IL-10, IL-12, IL-13, and IFN- $\gamma$, corresponding to cytokines with a mixed 
Table 1. Levels of Cytokines (PG/Ml) Accumulated over 7 Days in Bulk Cell Culture Supernatants of cDCs Versus SMART-DCs

\begin{tabular}{lcc}
\hline Cytokine & $c D C$ & SMART-DC \\
\hline IL1b & $92.8 \pm 77.6$ & $344.8 \pm 207.6^{*}$ \\
IL2 & $4.3 \pm 1.6$ & $3.0 \pm 0.2$ \\
IL5 & $3.2 \pm 0.0$ & $3.2 \pm 0.0$ \\
IL6 & $413.0 \pm 342.9$ & $1003.9 \pm 611.1^{*}$ \\
IL7 & $20.5 \pm 11.7$ & $31.3 \pm 10.6$ \\
IL8 & $2057.0 \pm 1069.5$ & $5321.0 \pm 2032.0$ \\
IL10 & $46.5 \pm 24.5$ & $22.3 \pm 9.8$ \\
IL12 & $5.8 \pm 2.0$ & $7.5 \pm 2.2$ \\
IL13 & $8.7 \pm 0.4$ & $9.9 \pm 0.9$ \\
IFNy & $6.1 \pm 2.0$ & $11.1 \pm 1.0$ \\
MCP-1 & $502.7 \pm 253.6$ & $3435.7 \pm 842.5^{*}$ \\
TNFa & $268.4 \pm 164.2$ & $564.9 \pm 222.4^{*}$ \\
\hline
\end{tabular}

Data is shown as average of triplicate independent experiments \pm standard deviation. ${ }^{*} \mathrm{p}<0.05$.

Th1/Th2 pattern (Table 1), which may reflect the downstream effects of GM-CSF (expressed typically in Th1 cells) and IL-4 (expressed typically in Th2 cells) in the genetic programming of the SMART-DCs.

Human monocyte-derived SMART-DCs readily engraft and show high viability after injection in Nod.SCID ${ }^{-1-}$ .$I L 2 r \gamma^{-/-}$and Nod.Rag1 ${ }^{-/-} . I L 2 r \gamma^{-/-}$mice

The engraftment and viability of SMART-DCs was tracked in vivo using noninvasive optical bioluminescence imaging analysis. We labeled the cells for co-expression of the reporter gene luciferase by co-transduction with LV-fLUC. The production of bioluminescence after enzymatic catalyses of the substrate luciferin depends on ATP, and therefore bioluminescence emission by transduced cells is associated with an active cell metabolism and viability. Control monocytes / fLUC, cDC-fLUC, or SMART-DCs-fLUC $\left(5 \times 10^{5}\right.$ cells $)$ were injected subcutaneously in the hind flank of NOD/RAG1 ${ }^{-/-}$ $/ \mathrm{IL} 2 \mathrm{r} \gamma^{-/-}$(NRG; Fig. 3) or NOD-SCID/IL2 $\mathrm{r} \gamma^{-/-}$(NOG) immunodeficient mice (Supplementary Fig. S3a) and the kinetics of the bioluminescence signal on the injection sites was followed noninvasively by optical imaging for 30 days. Mice injected with control monocytes or cDCs marked with luciferase showed very low levels of bioluminescence signal on

FIG. 3. In vivo engraftment and viability of SMARTDCs injected subcutaneously into Nod.Rag1-/-.IL2r $\gamma^{-/-}$ mice. (a) Experimental design to assess in vivo engraftment and viability of SMART-DCs. (b) In vivo optical imaging analysis of the injection sites obtained from representative Nod.Rag1 $1^{-/-}$.IL2 $\mathrm{r}^{-/-}$(NRG) mice administered subcutaneously with monocytes (Mono), cDCs, or SMART-DCs marked with firefly luciferase (fLUC). The reference color bar indicates high levels of bioluminescence signal in red and low levels in blue. (c) The bioluminescence signal emitted from sites of administration (or regions of interest) of monocytes (white bars), cDCs (diagonal-patterned bars), and SMART-DCs (black bars) was quantified and is represented as average ( $n=4$ to 5 mice per group). Error bars indicate standard error of mean (SEM). ${ }^{*} p<0.05,{ }^{* *} p<0.01$. the injection sites. In sharp contrast, SMART-DCs injected into mice immediately after transduction showed high levels of bioluminescence signal, lasting for 30 days (Fig. 3, Supplementary Fig. S3a). Notably, SMART-DCs /fLUC that were maintained for 7 days in culture prior to subcutaneous administration showed drastically reduced engraftment and lacked long-term viability (Supplementary Fig. S3b). All together, these results recapitulated in a xenogeneic mouse model our previous observation that SMART-DCs administered subcutaneously shortly after lentiviral transduction are highly viable in vivo.

a

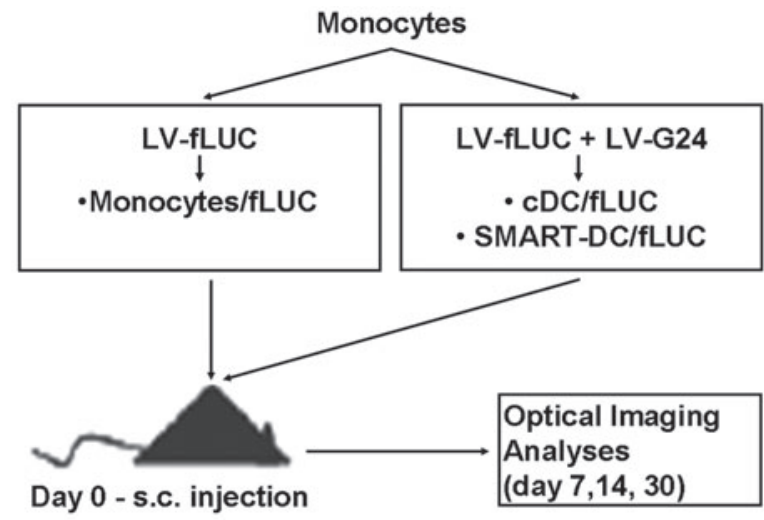

b

\section{$7 d$}

$14 d$

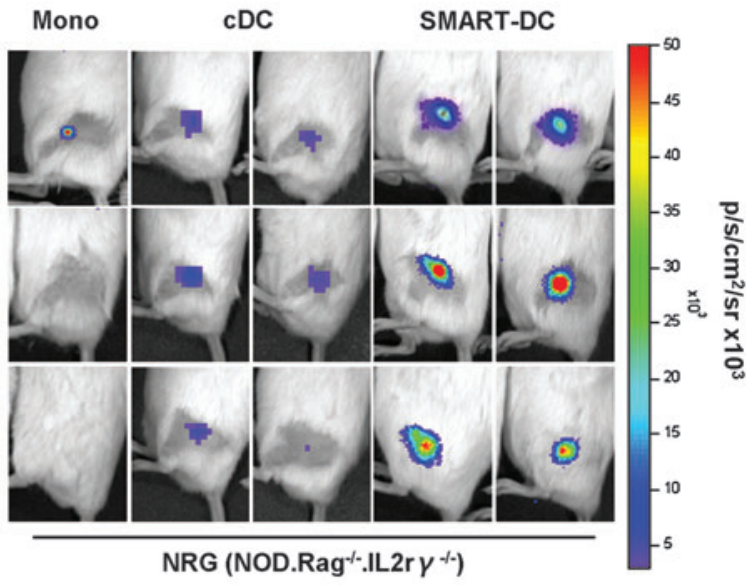

C

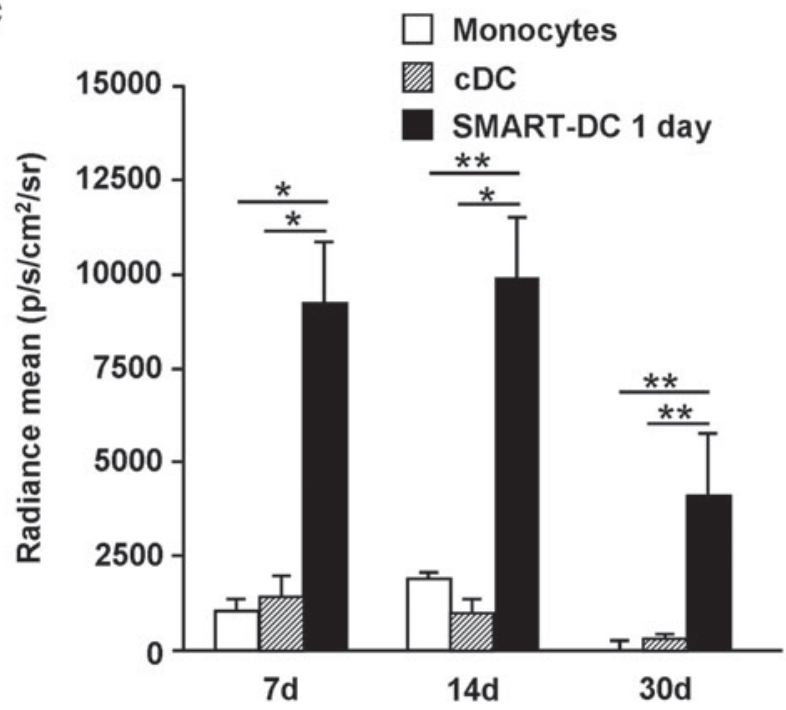




\section{Human SMART-DCs enhance the expansion} and co-localize with autologous $T$ cells in vivo

The preconditioning effects of human SMART-DCs on the expansion and biodistribution of autologous T cells in NRG mice were evaluated in vivo by monitoring the human $\mathrm{T}$ cells by optical imaging analyses. For transduction of human $\mathrm{T}$ cells with luciferase, $\mathrm{CD} 14^{-}$cells were isolated and cultured for $48 \mathrm{hr}$ in the presence of bead-conjugated antibodies (CD2/CD3/CD28) supplemented with cytokines (IL-7 and IL-15) because pre-activation of $\mathrm{T}$ lymphocytes is a prerequisite for efficient lentivirus-mediated gene transfer (Korin and Zack, 1998; Maurice et al., 2002). Transduced T cells showed high luciferase activity (Supplementary Fig. S4) and flow cytometric analysis of GFP expression after transduction of T cells with LV-GFP showed approximately 65\% GFP-positive cells in both $\mathrm{CD}^{+} / \mathrm{CD}^{+}$and $\mathrm{CD}^{+} / \mathrm{CD}^{+}$ populations (Supplementary Fig. S4), which did not affect the $\mathrm{CD}^{+}: \mathrm{CD}^{+} \mathrm{T}$ cell ratio (approximately 3:1).

NRG mice were preconditioned with $5 \times 10^{5}$ SMART-DCs or SMART-DCs-pp65 subcutaneously on the left hind flank on day -7 (mice injected with PBS served as controls). On day $0,5 \times 10^{6}$ autologous T/fLUC cells were injected intravenously and in vivo bioluminescence imaging analyses were performed on days 7, 14, and 30 (Fig. 4a). Bioluminescence detected on the flanks, corresponding to the injection site of SMART-DCs was 10- to 100-fold higher than for PBS control mice at day 7 (Fig. 4b and c) Mice preconditioned with SMART-DCs also showed significantly higher levels of luminescence signals in the spleen compared with PBS controls (Fig. $4 \mathrm{~b}$ and d). Preconditioning the mice with SMART-DCs expressing pp65 consistently resulted in even higher levels of bioluminescence, reflecting higher recruitment of $\mathrm{T}$ cells to the injection sites than with SMART-DCs lacking the antigen (Fig. 4b-d). All together, these dynamic optical imaging observations demonstrated the capacity of the SMART-DCs, and even more so for the SMART-DCs-pp65, to recruit T cells to the injection sites, where they persisted for several weeks.

Cryosections of spleens collected from mice on day 30 were analyzed by immunofluorescence microscopy for the in situ detection of cytotoxic T cell $\left(\mathrm{CTL}, \mathrm{CD} 8^{+}\right)$, helper $\mathrm{T}$ cell $\left(\mathrm{Th}, \mathrm{CD} 4^{+}\right)$, and endothelial cell $\left(\mathrm{CD} 31^{+}\right)$markers. Mice implanted with SMART-DCs and with SMART-DC-pp65 showed conspicuous distribution of large cellular clusters in the spleen (Fig. 4e). The clusters formed preponderantly around sites of vascularization (CD31 stained), with $\mathrm{CD} 8^{+}$ $\mathrm{T}$ cells in the center and $\mathrm{CD} 4^{+} \mathrm{T}$ cells in the periphery.

\section{Rapid early expansion of human $C D 8^{+} T$ cells in mice implanted with SMART-DC-pp65}

Expansion of human lymphocytes in the blood and spleen of NRG mice was evaluated on day 30 after infusion by flow cytometry analyses. In line with the observations obtained by in vivo imaging, SMART-DCs and SMART-DC-pp65 preconditioning was associated with a higher expansion of human $\mathrm{CD} 45^{+}$cells in mouse peripheral blood and spleen on day 30 (approximately $25 \%$ of the cells were of human origin in comparison with $6 \%$ for PBS control mice, $p<0.05$ ) (Fig. 5a and $b$ ).

Kinetic analyses of the frequency of human $\mathrm{CD} 3^{+} / \mathrm{CD}^{+}$ and $\mathrm{CD}^{+} / \mathrm{CD}^{+} \mathrm{T}$ cells in the peripheral blood were performed by sequential blood sampling on days 7,14 , and 30 after T-cell infusion. Compared with controls, mice preconditioned with SMART-DCs showed a significant increase in the frequency of circulating human $\mathrm{CD}^{+} / \mathrm{CD}^{+} \mathrm{T}$ cells throughout the time of analysis (Fig. 5c). However, SMARTDC-pp65 preconditioning induced a more dramatic increase in frequencies of circulating human $\mathrm{CD}^{+} / \mathrm{CD}^{+} \mathrm{T}$ cells in mice than SMART-DCs (2.1-fold increase on day $7, p<0.05$; 2.5 -fold increase on day 30, $p<0.05$ ) (Fig. 5c). Seven days after T-cell infusion, we detected on average approximately $1.5 \%$ of human $\mathrm{CD}^{+} / \mathrm{CD}^{+}$circulating $\mathrm{T}$ cells in the peripheral blood of SMART-DC-pp65 preconditioned mice. This frequency increased gradually, and 30 days later, approximately $5 \%$ of the mouse circulating peripheral blood cells were human CTLs.

The frequency of $\mathrm{CD}^{+} / \mathrm{CD}^{+}$human $\mathrm{T}$ cells was also significantly increased by preconditioning mice with SMART-DCs (day 7: $1.31 \%$ for SMART-DCs vs. $0.21 \%$ for control, $p<0.05)$, but this effect was more noticeable on day 30 , when the frequency of human $\mathrm{CD}^{+} / \mathrm{CD}^{+} \mathrm{T}$ cells corresponded to almost $20 \%$ of the mouse circulating peripheral blood leukocytes (PBLs; Fig. 5d). Notably, only at early time points (on day 7) SMART-DC-pp65 preconditioning resulted in significantly higher expansion of $\mathrm{CD}^{+} / \mathrm{CD} 8^{+}$and $\mathrm{CD}^{+} / \mathrm{CD}^{+}$human $\mathrm{T}$ cells compared with preconditioning with SMART-DCs (Fig. 5c and d). Overall, preconditioning mice with SMART-DCs seemed to favor CD4 ${ }^{+}$T-cell expansion, whereas preconditioning with SMART-DCs-pp65 generated faster expansion of both $\mathrm{CD} 8^{+}$and $\mathrm{CD} 4^{+} \mathrm{T}$ cells.

We subsequently analyzed the frequencies of effector memory $\left(\mathrm{CD} 45 \mathrm{RA}^{-} / \mathrm{CCR}^{-}\right)$versus naïve $\left(\mathrm{CD}^{-} 5 \mathrm{RA}{ }^{+} /\right.$ $\mathrm{CCR}^{+}$) human $\mathrm{CD} 8^{+} \mathrm{T}$ cells that engrafted and repopulated spleens of mice $(n=3) 30$ days after lymphocyte infusion. Significantly higher levels of effector memory $\mathrm{CD}^{+} / \mathrm{CD}^{+}$ $\mathrm{T}$ cells and lower levels of naïve $\mathrm{CD}^{+} / \mathrm{CD}^{+} \mathrm{T}$ cells were observed when mice were immunized with SMART-DCs or SMART-DCs-pp65 compared with control mice (Fig. 5e and f). Regarding the phenotype of $\mathrm{CD}^{+} / \mathrm{CD}^{+}{ }^{+} \mathrm{T}$ cells at this late time-point we observed in all the groups that approximately $90 \%$ of the analyzed cells corresponded to effector memory cells (Fig. 5g). The naïve $\mathrm{CD}^{+} / \mathrm{CD}^{+}$phenotype was observed in less than $10 \%$ of analyzed cells, regardless of the immunization (Fig. 5h). Although within the experimental period of analyses (30 days after T-cell infusion) we did not observed clinical signs of graft vs. host disease (GvHD), it is possible that the conversion of naïve into effector memory phenotype of the $\mathrm{CD} 4^{+} \mathrm{T}$ cells could rather indicate the onset of xenogenic GvHD as reported previously by other groups (Yamashita et al., 2004).

\section{Activation of pp65-reactive $T$ cells after in vitro stimulation with $D C s$}

We next examined whether the faster expansion of CD8 ${ }^{+}$ CTLs caused by preconditioning SMART-DCs-pp65 in vivo was associated with anti-pp65 antigenic responses. T cells obtained from a CMV sero-positive donor with pre-existing HLA-A*0201 and $-\mathrm{B}^{*} 0702$ restricted responses to pp65 were enriched by $\mathrm{CD}^{+}$or $\mathrm{CD}^{+}$selection and primed once with autologous DCs (Supplementary Fig. S5a). Control cDCs and cDCs loaded with a pp65 peptide pool were compared with SMART-DCs-pp65. Notably, we were able to detect high HLA-A*0201 and HLA-B*0702 restricted pp65 reactivity after 

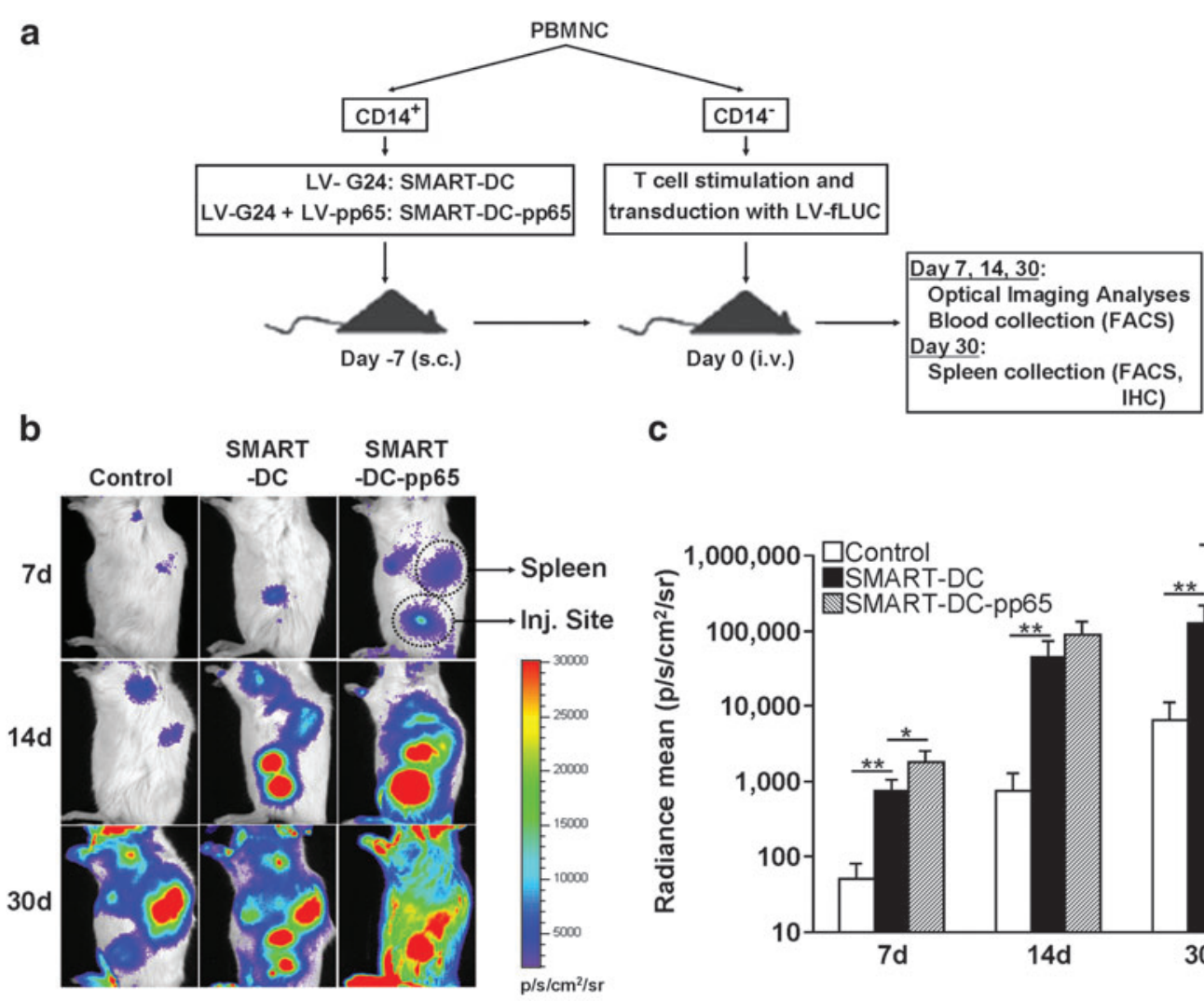

C IHC)

d

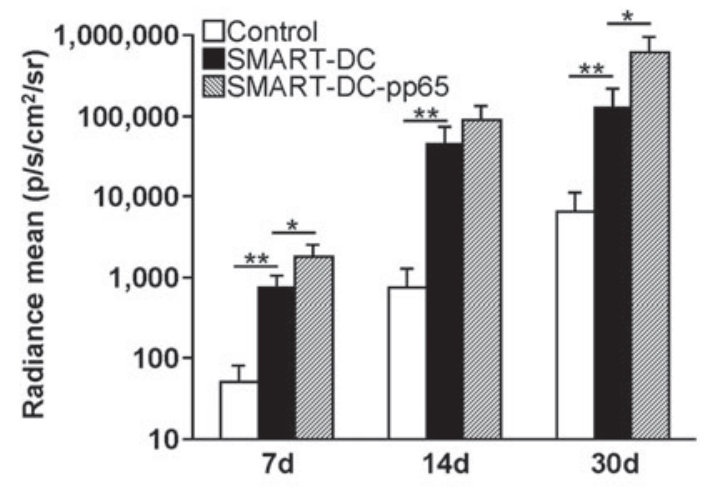

\section{e}

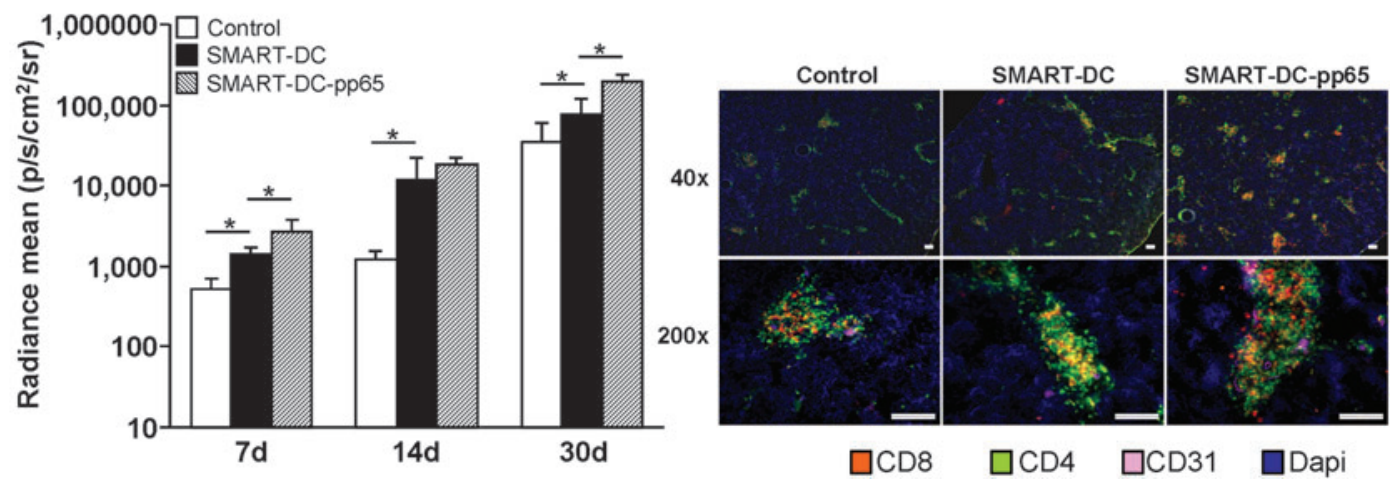

FIG. 4. Biodistribution analyses of autologous human lymphocytes in NRG mice preconditioned with SMART-DCs. (a) Schematic representation of human T-cell expansion experiments. Control untreated mice or mice preconditioned with a single injection of SMART-DCs or SMART-DCs-pp65 (on day -7) were subsequently infused intravenously with autologous human T cell expressing fLUC (on day 0). In vivo imaging analyses were performed on day 7, 14, and 30 after T-cell infusion. (b) In vivo optical imaging analysis showing biodistribution of human fLUC/T cells on days 7, 14, and 30. One representative NRG mouse per group is shown (untreated control or preconditioned with SMART-DCs or SMART-DCs-pp65). The bioluminescence signal detectable from the DC administration site (c) and spleen (d) was quantified and is represented as average for each group: control (white bars), SMART-DCs (black bars), and SMART-DCs-pp65 (diagonal-patterned bars). Error bars represent SEM. ${ }^{*} p<0.05$ and ${ }^{* *} p<0.01, n=5$ per group. (e) Cryosections of spleens obtained from control, SMARTDC, and SMART-DC-pp65 preconditioned mice (magnifications at $4 \times$ and $20 \times$ ), were analyzed by immunofluorescence stained with anti-human CD4 ${ }^{+}$(green), anti-human $\mathrm{CD}^{+}$(orange), and anti-mouse CD31 ${ }^{+}$(violet) and counterstained with DAPI (blue). Scale bars indicate $200 \mu \mathrm{m}$.

a single stimulation with both DCs and SMART-DCs when we used isolated $\mathrm{CD}^{+} \mathrm{T}$ cells for co-culture but not when we used bulk $\mathrm{CD}^{+} \mathrm{T}$ cells (Supplementary Fig. S5b). In contrast to CDCs, which required maturation when co-cultured with $\mathrm{CD}^{+} \mathrm{T}$ cells for stimulation of antigen-specific responses, SMART-DCs-pp65 were less dependent on maturation with exogenously added factors in order to stimulate anti-pp65 CD8 ${ }^{+} \mathrm{T}$-cell responses. Incidentally, the frequency of regulatory $\mathrm{T}$ cells (or Tregs, defined as CD $4^{+} / \mathrm{CD} 25^{\text {high }} /$ $\mathrm{CD}^{2} 7^{-}$cells) after stimulation of $\mathrm{T}$ cells with cDCs or 


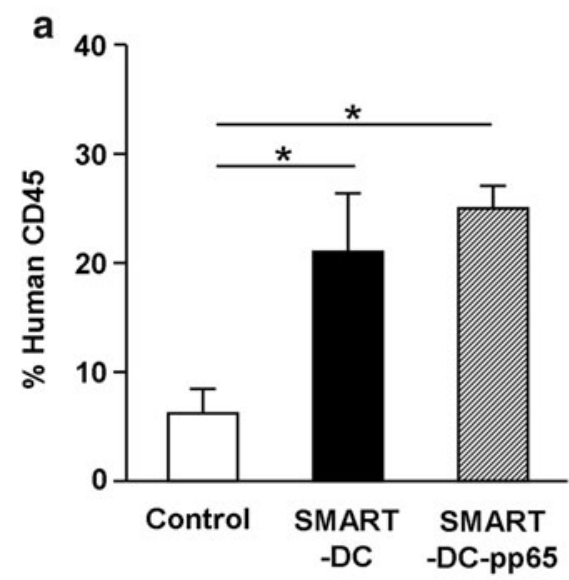

C

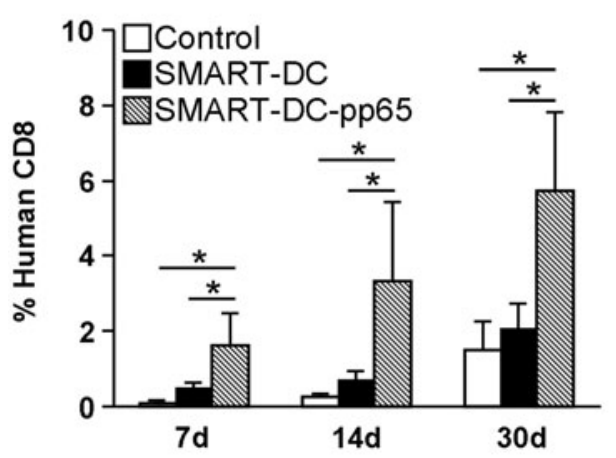

e

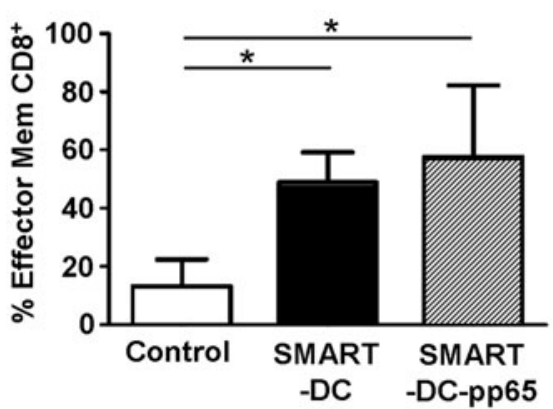

g

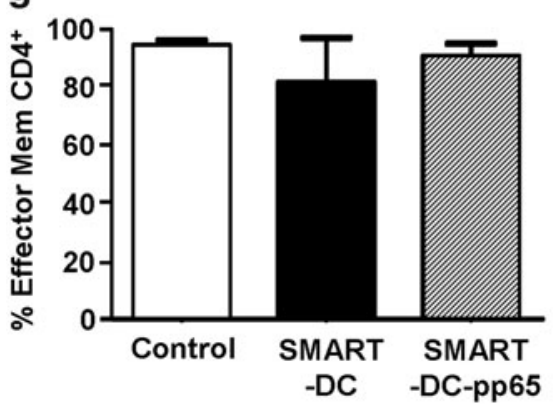

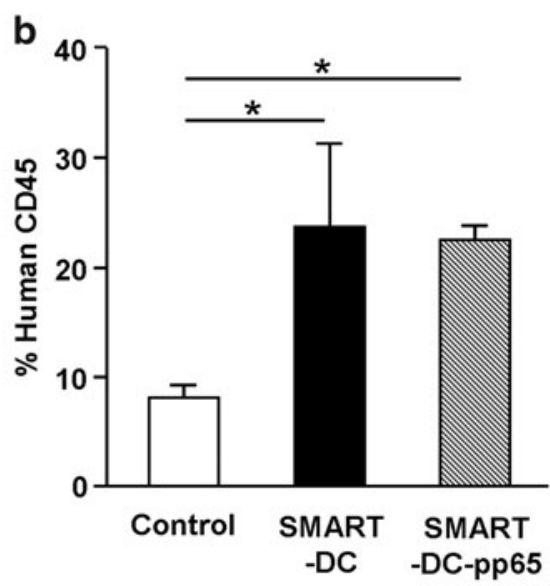

d

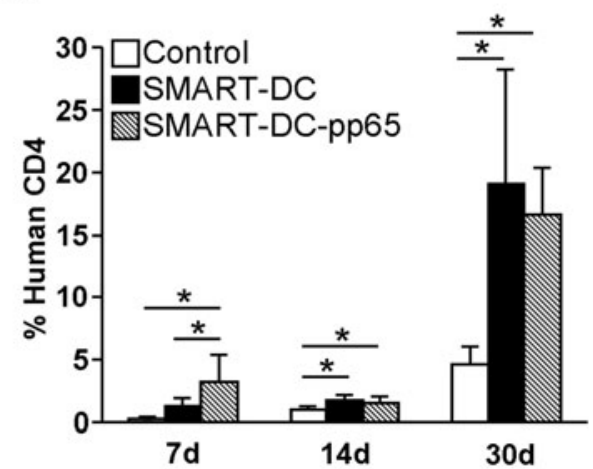

f

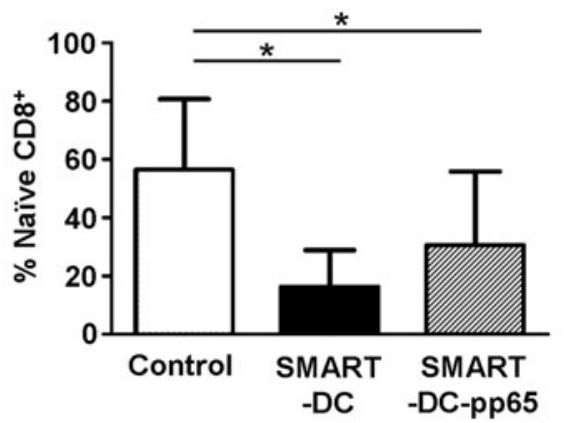

h

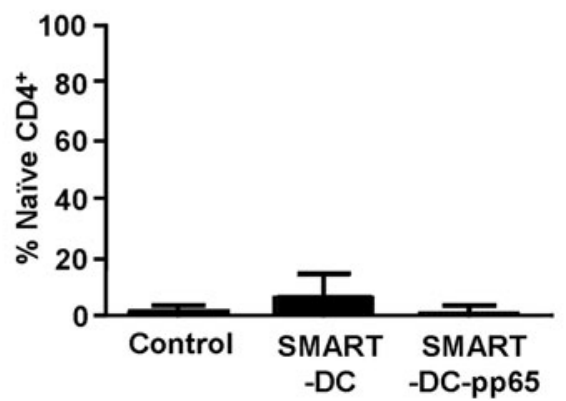

FIG. 5. Flow cytometry analyses of expanded human $\mathrm{T}$ cells in peripheral blood and spleen of NRG mice preconditioned with SMART-DCs. Frequency of human $\mathrm{CD} 45^{+}$cells in peripheral blood (a) and spleen (b) from control (white bars), SMART-DC (black bars) and SMART-DC-pp65 (diagonalpatterned bars) preconditioned mice, as analyzed by flow cytometry on day 30. Frequency of human $\mathrm{CD}^{+} / \mathrm{CD}^{+}{ }^{+} \mathrm{T}$ cells $(\mathrm{c})$, human $\mathrm{CD}^{+} / \mathrm{CD}^{+}{ }^{+} \mathrm{T}$ cells (d) in peripheral blood from control, SMART-DC, and SMARTDC-pp65 preconditioned mice analyzed on days 7, 14, and 30 . Frequencies of human naïve $\mathrm{CD}^{+} / \mathrm{CD} 45 \mathrm{RA}^{+} / \mathrm{CCR}^{+}$(e), effector memory (Mem) $\mathrm{CD}^{+} /$ $\mathrm{CD}^{2} 5 \mathrm{RA}^{-} / \mathrm{CCR7}^{-}$(f), naïve $\mathrm{CD}^{+} / \mathrm{CD} 45 \mathrm{RA}^{+} / \mathrm{CCR}^{+} \quad$ (g), and effector memory $\mathrm{CD}^{+} /$ CD45RA ${ }^{-} / \mathrm{CCR7}^{-} \mathrm{T}$ cells (h) detectable in spleens from mice analyzed on day 30 in each group. Error bars represent SEM. ${ }^{*} p<0.05$ and $n=5$ per group.
SMART-DCs decreased in relation to nonstimulated controls (Supplementary Fig. S5c).

As expected, the frequency of effector memory $\mathrm{T}$ cells $\left(\mathrm{CD} 45 \mathrm{RA}^{-} / \mathrm{CD}^{-} \mathrm{L}^{-}\right)$increased upon co-culture of $\mathrm{CD}^{+} \mathrm{T}$ cells with DCs presenting the pp65 antigen (Supplementary
Fig. S6b), with concomitant reduction in the frequency of naïve $\mathrm{CD} 8{ }^{+} \mathrm{T}$ cells, whereas the central memory $\mathrm{CD} 8^{+} \mathrm{T}$-cell compartment remained less affected (Supplementary Fig. S6c). We did not observed variations in the frequency of central memory $\mathrm{CD}^{+} \mathrm{T}$ cells after co-culture with DCs 
loaded with peptide (Supplementary Fig. S6d), and these results might suggest a direct conversion of naïve into effector memory cells after antigen stimulation. On the other hand, culture of DCs with $\mathrm{CD}^{+}{ }^{+} \mathrm{T}$ cells resulted into more than $90 \%$ of the cells demonstrating effector memory CD4 ${ }^{+}$ T-cell phenotype (Supplementary Fig. S6d).

\section{SMART-DC-pp65 is a better APC for in vivo expansion and co-localization with $T$ cells at the injection sites compared with cDCs}

We compared the "gold standard" cDCs (not loaded or loaded with a pp65 overlapping peptide pool) with SMARTDCs (mock or internally loaded with the protein through LVpp65 co-transduction) regarding the in vivo T-cell stimulatory effects. For preconditioning, freshly transduced SMART-DCs were injected subcutaneously into the flanks of NRG mice on day -7 and $\mathrm{T} / \mathrm{fLUC}$ cells were injected intravenously on day 0 (Fig. 6a). Ex vivo differentiated cDCs were injected subcutaneously on day 0 , on the same day that T/fLUC cells were injected intravenously (Fig. 6a). Expansion and biodistribution of the T/fLUC cells were assessed by optical imaging analyses (Fig. 6b-d). Bioluminescence signal consistently colocalized with the sites of SMART-DC and SMART-DC-pp65 injections, whereas the signal was hardly detectable at sites injected with cDCs or cDCs-pp65 (Fig. 6c). Mice preconditioned with SMART-DC-pp65 also showed significantly higher luminescence signals in spleen compared with the cDC-pp65 injected group (on day 7: 2.7-fold higher, $p<0.05$; Fig. 6d).

\section{SMART-DCs-pp65 promote higher $C D 8^{+}$and $C D 4^{+}$ $T$-cell expansions in mice than conventional DCs pulsed with a pp65 peptide pool}

In order to characterize the T-cell populations stimulated and expanded in vivo after preconditioning with cDCs (mock or pp65 peptide pulsed) or SMART-DCs (mock or LV-pp65 transduced), peripheral blood was collected on days 7 and 14 after $\mathrm{T} / \mathrm{fLUC}$ infusions. The highest frequency of circulating human $\mathrm{CD}^{+} \mathrm{CTLs}$ was observed in mice preconditioned with SMART-DCs-pp65, which amounted to approximately $1 \%$ and $2 \%$ of the PBLs on days 7 and 14, respectively (Fig. 7a). In vivo preconditioning with cDCs pulsed with pp65 peptides prior to T-cell infusion resulted in significantly lower numbers of human CD8 ${ }^{+}$CTLs than with SMARTDCs-pp65. Confirming our previous results, SMART-DCs lacking the pp65 antigen also promoted steady expansion of human CD8 ${ }^{+}$CTLs in vivo (Fig. 7a). The expansion of human $\mathrm{CD}^{+} \mathrm{T}$ cells was also significantly increased at day 14 after SMART-DC-pp65 preconditioning (Fig. 7b). In contrast, cDCs pulsed with pp65 peptides produced only modest levels of $\mathrm{CD}^{+}{ }^{+} \mathrm{T}$-cell expansion in vivo (Fig. $7 \mathrm{~b}$ ).

\section{SMART-DCs-pp65 promote more consistent multiantigenic activation of pp65-specific $C D 8^{+} T$ cells in vivo than $c D C s$ pulsed with a pp65 peptide pool}

Since the previous observations indicated that preconditioning with SMART-DCs-pp65 might particularly enhance engraftment and stimulation of $\mathrm{CD}^{+} \mathrm{T}$ cells, we performed experiments to measure the pp65-specific immune responses in mice infused with non-immunereactivated autologous $\mathrm{CD}^{-} 4^{-}$lymphocytes or $\mathrm{CD}^{+} / \mathrm{CD} 8^{+}$ selected T cells. NRG mice ( $n=2$ per group) were preconditioned with cDCs pulsed with the pp65 peptide pool (on day 0 ) or preconditioned with SMART-DCs-pp65 (on day -7). IFN- $\gamma$-ELISPOT analysis was used to evaluate the functionality of the $\mathrm{CD} 8^{+} \mathrm{T}$ cells expanded in vivo in NRG mice. For this assay, spleens were collected on day 10 after the infusion of lymphocytes and disrupted into single-cell suspensions, and human $\mathrm{CD}^{+} \mathrm{T}$ cells were sorted by flow cytometry. Selected human $\mathrm{CD}^{+}$cells were restimulated ex vivo with recall antigenic pool peptides corresponding to CMV, EpsteinBarr virus, and influenza virus epitopes (CEF) or with the pp65 peptide pool and analyzed by ELISPOT for IFN- $\gamma$ production. The assay proved to be specific and valid because the negative control wells (not restimulated) showed no reactivity, whereas the positive control wells (restimulated with CEF recall antigen) showed uniform reactivity (Fig. 7c). The test groups (restimulated with pp65 peptide pool) showed differential amount and intensity of spots when $\mathrm{T}$ cells were obtained from mice administered with conventional DCs/ pp65 or SMART-DCs-pp65 (Fig. 7c). Mice infused with CD14- lymphocytes showed significantly higher pp65 reactivity after preconditioning with SMART-DC-pp65, than mice preconditioned with pp65-loaded cDCs (Fig. 7d). In mice infused with $\mathrm{CD}^{+} / \mathrm{CD}^{+}$-selected human $\mathrm{T}$ cells, the anti-pp65 reactivity was even higher (Fig. 7d).

Peripheral blood collected 10 days after the T-cell infusion was also analyzed for pp65-specific tetramer reactivity against $\mathrm{CD} 3^{+} / \mathrm{CD} 8^{+}$human $\mathrm{T}$ cells. Mice preconditioned with pp65-pulsed cDCs and later infused with CD14 ${ }^{-}$lymphocytes produced high frequencies of pp65-reactive $\mathrm{T}$ cells that were either $\mathrm{A}^{*} 0201(12.5 \%)$ or $\mathrm{B}^{*} 0702$ restricted $(4.8 \%)$ (Table 2). One of two mice preconditioned with SMARTDCs-pp65 and later infused with CD14- lymphocytes contained high frequency of $\mathrm{T}$ cells with pp65 reactivity against both $\mathrm{A}^{*} 0201(5.9 \%)$ and $\mathrm{B}^{*} 0702(16.7 \%)$ tetramers, demonstrating response to pp65 epitopes presented by different HLAs. Concurrently, both mice preconditioned with pp65pulsed $\mathrm{CDC}$ and infused with selected $\mathrm{CD}^{+} / \mathrm{CD} 8^{+} \mathrm{T}$ cells had $C D 8^{+} T$ cells reactive against the $B^{*} 0702$ tetramer, but no reactivity was seen against the $A^{*} 0201$ tetramer (Table 2). On the other hand, both mice preconditioned with SMART-DCpp65DC and infused with selected $\mathrm{CD}^{+} / \mathrm{CD}^{+} \mathrm{T}$ cells showed a high frequency of $\mathrm{T}$ cells in peripheral blood reactive against both $\mathrm{A}^{*} 0201(0.6 \%-3.9 \%)$ and the $\mathrm{B}^{*} 0702$ $(2.9 \%-6.2 \%)$ tetramer (Table 2$)$.

Finally, we tested whether administration of SMARTDCs-pp65 needed to be performed prior to or could be administered concurrently with T cells for an optimal anti-pp65 immunization effect. For this comparison, nonstimulated lymphocytes were infused intravenously and 10 days after, mouse peripheral blood was collected and frequencies of human $\mathrm{T}$ cells were analyzed by flow cytometry. For all parameters analyzed (expansion of $\mathrm{CD} 4^{+} \mathrm{T}$ cells and $\mathrm{CD} 8^{+}$ $\mathrm{T}$ cells, and reactivity against pp65 measured by ELISPOT and tetramer analyses), the best results were obtained when NRG mice $(n=3)$ were injected with SMART-DCs-pp65 7 days prior to $\mathrm{T}$ cells, instead of concurrently (Fig. 8a-d). Monocytes or cDCs transduced with LV-pp65 or cDCs loaded with pp65 peptides and administered prior to T cells resulted into lower $\mathrm{CD}^{+}$and $\mathrm{CD} 8^{+} \mathrm{T}$-cell expansion than SMART-DCs-pp65 used for in vivo stimulation (Fig. 8a and b). Reactivity against pp65 was consistently superior when 
a

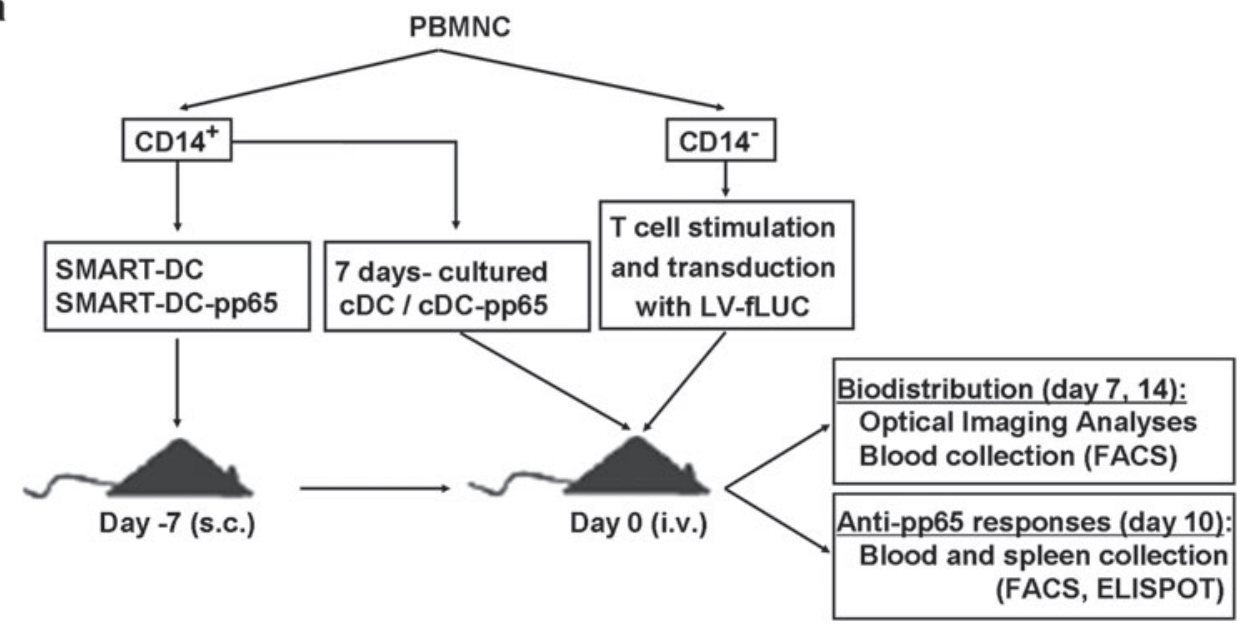

b

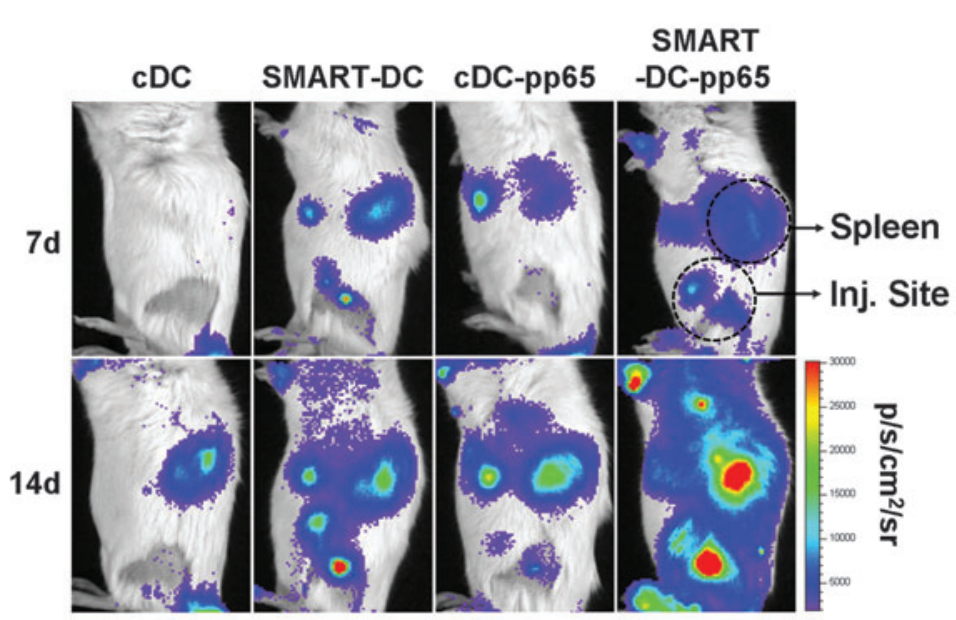

C

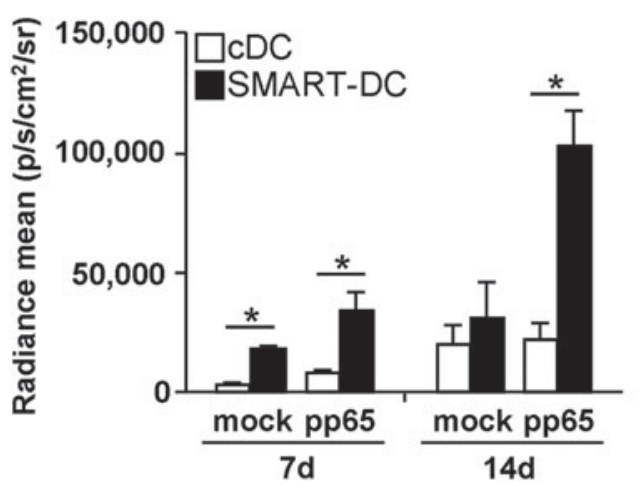

d

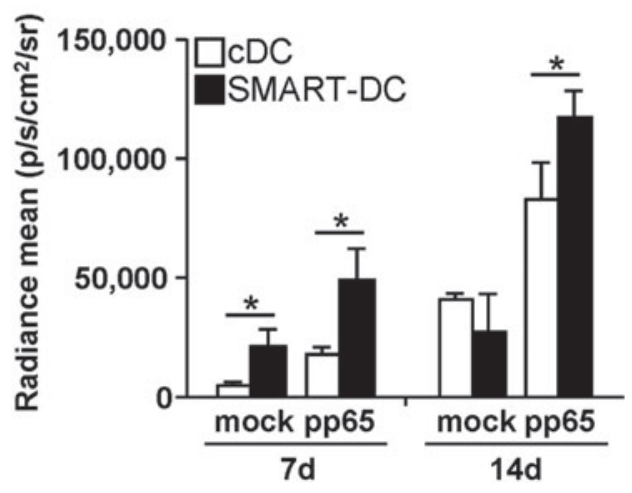

FIG. 6. T-cell engraftment, biodistribution, and persistency in NRG mice when co-administered with cDCspp65 versus preconditioned with SMART-DCs-pp65. (a) Schematic representation of the different treatment groups and treatment schedules: mice were preconditioned with SMART-DCs or SMART-DCs-pp65 on day -7 ; cDCs were generated on day -7 , differentiated in culture for 6 days and pulsed overnight with an overlapping pp65 peptide pool (cDC-pp65) or not pulsed (cDC) and transfer to NSG mice on day 0. Autologous human $\mathrm{T}$ cells expressing luciferase (fLUC/T Cells) were intravenously infused on day 0 . In vivo imaging analyses were performed on days 7 and 14 after T-cell infusion. (b) In vivo optical imaging analysis showing biodistribution of fLUC/T cells on days 7 and 14 in representative NRG mice for each group. Histogram bars represent the quantified bioluminescence detected in the injection site (c) and spleen (d) of mice for each group: "mock" represents control DCs not loaded with pp65 antigen; cDCs (white bars) and SMART-DCs (black bars) are compared. Error bars represent SEM. ${ }^{*} p<0.05$, $n=5$ per group.
SMART-DCs-pp65 were used as preconditioning APCs (Fig. 8c and d). SMART-DCs-pp65 administered on the same day as T cells resulted in lower T-cell stimulation in vivo (Fig. 8c and d).

\section{Discussion}

DCs are a rare population in the peripheral blood (less than $1 \%$ ) and the steady-state DC half-life lasts from days to a few weeks (Merad and Manz, 2009). Thus, we hypothesized that preconditioning an immune-deficient mouse host with long-lived human SMART-DCs conferring multiple human cytokine and potent antigenic stimulation would enhance the homeostatic and antigen-specific expansion of human $\mathrm{T}$ cells.

Several groups have reported the administration of human CDCs at the same time or after autologous T-cell infusion into immunodeficient mice as an approach to increase T-cell expansion, and in some cases stimulate antigenspecific responses (Supplementary Table S1). SCID or NOD/ SCID mice injected with PBMCs and then primed/boosted with DCs loaded with inactivated HIV (Yoshida et al., 2003; Lapenta et al., 2006) or with DCs transduced with herpes virus expressing HIVgp120 (Gorantla et al., 2005) showed immune reactivity against HIV, but the kinetics of T-cell 


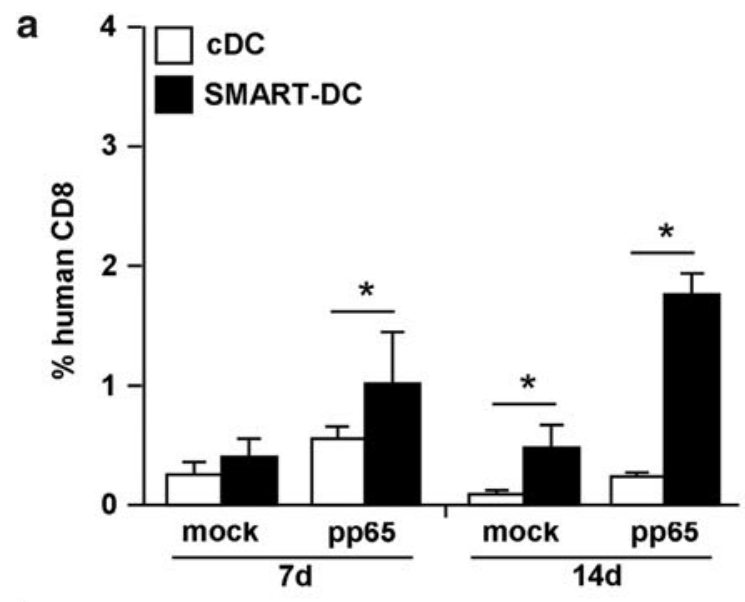

b

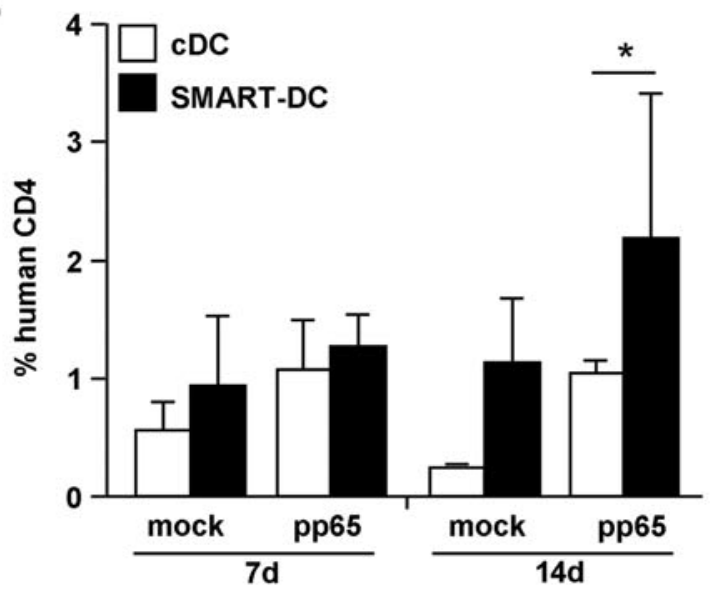

C
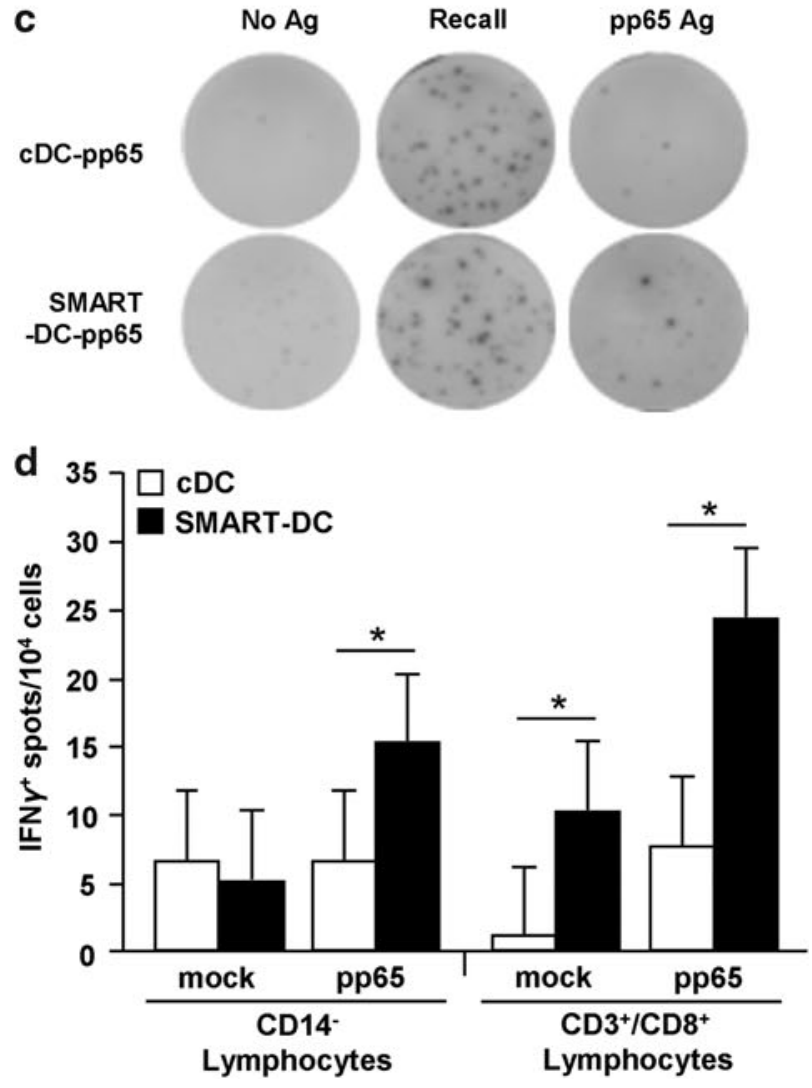

Table 2. Detection of pp65-Reactive Human T Cells Recovered from Mouse Peripheral Blood and Analyzed by Tetramer Assay

\begin{tabular}{|c|c|c|c|}
\hline $\begin{array}{l}\text { Injected } \\
\text { Lymphocytes }\end{array}$ & $A P C$ & $\begin{array}{c}A^{*} 0201 \\
(\% C D 3 / \\
C D 8)\end{array}$ & $\begin{array}{c}B^{*} 0702 \\
(\% C D 3 / \\
C D 8)\end{array}$ \\
\hline \multirow[t]{4}{*}{ CD14- } & cDC-pp65 \#1 & 0 & 4.8 \\
\hline & cDC-pp65 \#2 & 12.5 & 0 \\
\hline & SMART-DC-pp65 \#1 & 0 & 0 \\
\hline & SMART-DC-pp65 \#2 & 5.9 & 16.7 \\
\hline \multirow{4}{*}{$\mathrm{CD}^{+} / \mathrm{CD}^{+}$} & cDC-pp65 \#1 & 0 & 6.5 \\
\hline & cDC-pp65 \#2 & 0 & 10.6 \\
\hline & SMART-DC-pp65 \#1 & 3.9 & 2.9 \\
\hline & SMART-DC-pp65 \#2 & 0.6 & 6.2 \\
\hline
\end{tabular}

expansion and biodistribution was not described. A recent report employing the NOD/Shi-scid/IL $2 \gamma c^{\text {null }}$ (NOG) mouse model documented the use of DCs grown ex vivo to prime/ boost T-cell expansion, resulting into antigen-specific CD8 ${ }^{+}$ T-cell responses (CMVpp65, MART-1, and WT1), but in this case, explanted splenocytes were subsequently maintained and re-stimulated in vitro for measuring immune responses. A recent study using NOD/SCID/IL $2 \gamma c^{\text {null }}$ mice has reported successful expansion of adenovirus-specific human T lymphocytes by in vivo priming with monocyte-derived cDCs infected with adenoviral vectors (Harui et al., 2011). In this work, adenovirus-transduced cDCs were infused intraperitoneally concurrently and after $\mathrm{T}$ cells, and $\mathrm{T}$ cells were restimulated in vitro for measuring immune responses (Inoue et al., 2009).

In comparison with previous reports, we evaluated whether preconditioning the host with APCs enabling stable expression of several human cytokines and MHC class I and II could accelerate the engraftment of functional $\mathrm{CD}^{+}$and $\mathrm{CD}^{+} \mathrm{T}$ cells. The APCs, so-called SMART-DCs, consisted of lentivirally manipulated monocytes, which upon autonomous constitutive expression of GM-CSF and IL-4, resulted in a homogeneous DC population $\left(\mathrm{CD} 209^{+}, \mathrm{CD}^{+} 6^{+}\right.$, HLA$\mathrm{DR}^{+}$) expressing maturation markers such as CD83 and CD80. Since $20 \%$ of CMV-sero-positive adults have CD8 ${ }^{+} \mathrm{T}$ and $\mathrm{CD}^{+}{ }^{+} \mathrm{T}$ cells responding to pp65 immune stimulation (Sylwester et al., 2005), we used prescreened donors and

FIG. 7. Activation of pp65-specific CD8 ${ }^{+}$T-cell responses by SMART-DCs/pp65 in vivo. Frequency of (a) human $\mathrm{CD}^{+} / \mathrm{CD}^{+} \mathrm{T}$ cells and (b) human $\mathrm{CD} 3^{+} / \mathrm{CD} 4^{+} \mathrm{T}$ cells in peripheral blood obtained from mice preconditioned with mock or pp65 peptide pulsed cDCs (white bars) and mock or LV-pp65-co-transduced SMART-DCs (black bars); flow cytometry analyses were performed with blood collected on days 7 and 14 after T-cell infusion. Error bars represent SEM. ${ }^{*} p<0.05$ and $n=5$ per group. (c,d) IFN $\gamma$-ELISPOT assay was performed with human $\mathrm{CD}^{+}$cells recovered from spleens on day 10 after infusion of $\mathrm{CD}_{1} 4^{-}$or $\mathrm{CD} 3^{+} / \mathrm{CD} 8^{+}$lymphocytes in mice preconditioned with Conv-pp65 (white bars) or SMART-DC-pp65 (black bars). CD3 ${ }^{+}$cells were unstimulated (no $\mathrm{Ag}$ ) or restimulated with CEF peptide (recall) or pp65 pool peptide (pp65 Ag) overnight and further analyzed for IFN- $\gamma$ production. Averaged spot counts per $10^{4}$ cells are shown. Error bars indicate standard deviation (SD). ${ }^{*} p<0.05$. 
pp65 as a model system for comparing cDCs and SMARTDCs. CMV pp65 epitopes were loaded as a peptide pool (for in vitro experiments) or the full-length antigenic protein was co-expressed in SMART-DCs and used as a preconditioning regimen to stimulate antigen-specific $\mathrm{T}$-cell responses directly in vivo.

Preconditioning the mice with human SMART-DCs-pp65 was performed with a single subcutaneous injection 1 week prior to intravenous infusion with autologous $\mathrm{T}$ cells. We used lower amounts of $\mathrm{T}$ cells $\left(5 \times 10^{6}\right.$ cells $)$ than generally reported in previous studies (mostly $10^{7}$ cells and higher)
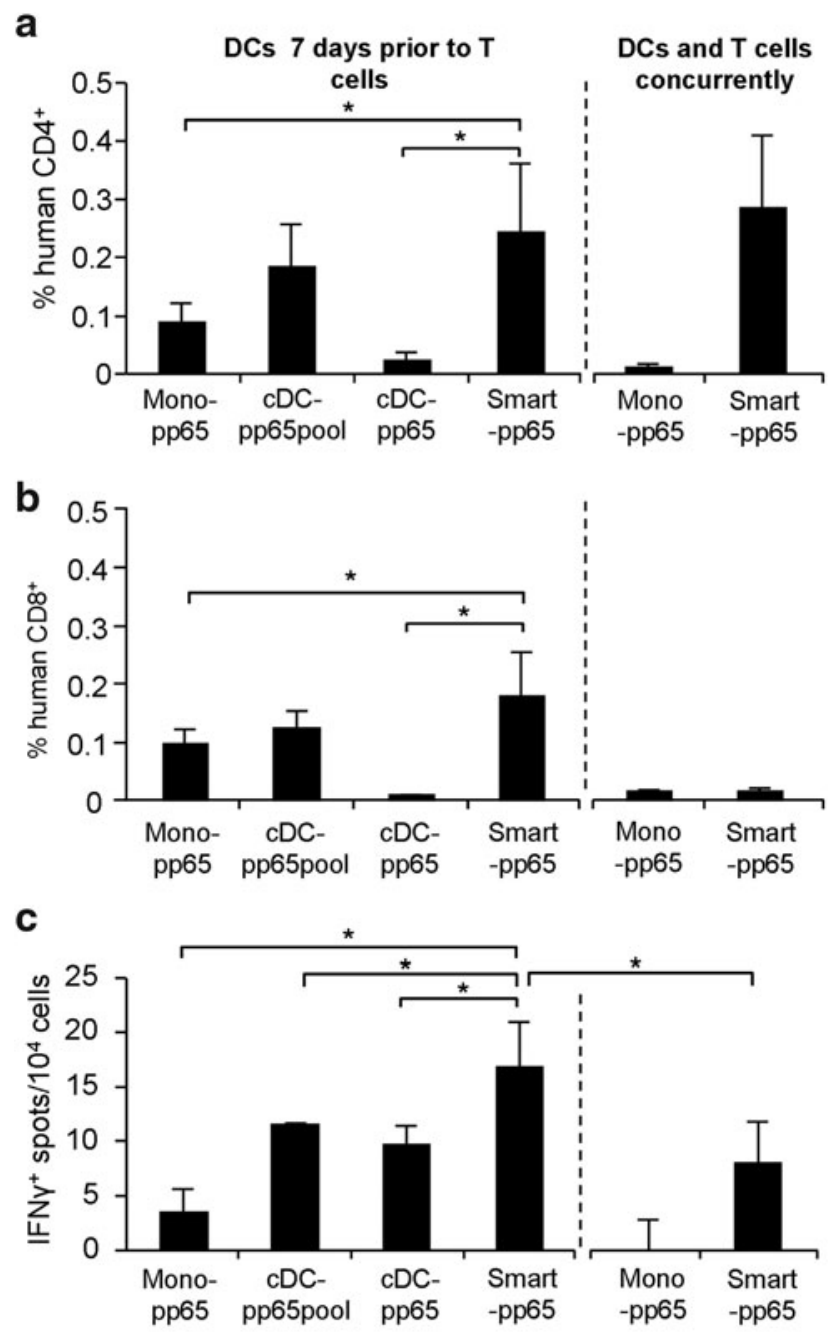

d

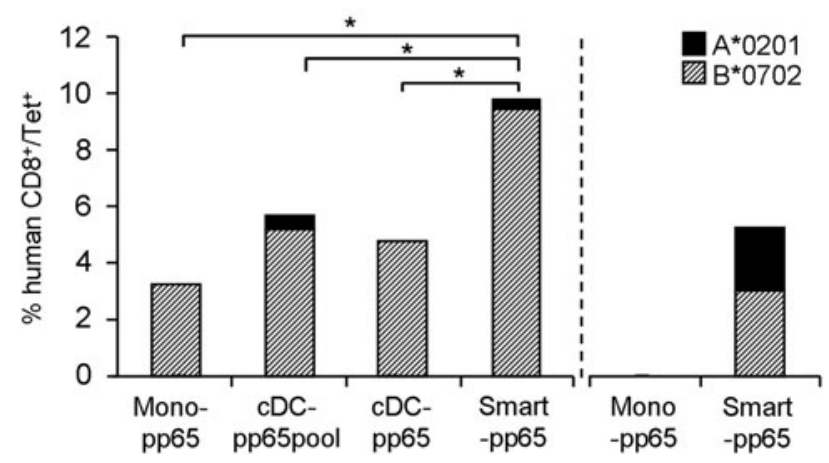

(Supplementary Table S1). We chose local subcutaneous delivery in the mouse, comparable to the route used in DC immunotherapy clinical trials in humans. Using noninvasive optical imaging analyses, we observed that SMART-DCs could be readily tracked on the injection site for at least 30 days. These results reproduced our prior observations reporting the viability and stability of mouse SMART-DCs administered subcutaneously in syngeneic C57BL/6 mice (Koya et al., 2007; Pincha et al., 2011).

The sequential biodistribution analyses of marked $\mathrm{T}$ cells conducted with optical imaging analyses were very critical to demonstrate the potency of SMART-DCs-pp65. One week after infusion, at least half of the $\mathrm{T}$ cells were already concentrated at the site of SMART-DC-pp65 administration and this co-localization lasted for 4 weeks, indicative of stable DC/T-cell immunologic interactions. Homing of the human $\mathrm{T}$ cells also occurred at early time points in the spleens (up to 14 days after T-cell infusion). Four weeks after T-cell infusion, approximately $25 \%$ of the cells in the mouse spleen consisted of human T cells, which formed noticeable cellular clusters along blood vessels. At later time points of our model (i.e., 14 days after T-cell infusion), $\mathrm{T}$ cells were distributed systemically in the whole body of the animal.

SMART-DCs co-expressing the pp65 antigen stimulated the fastest expansion of human $\mathrm{CD}^{+}$and $\mathrm{CD}^{+}{ }^{+}$T-cell numbers in vivo. This expansion was clearly noticeable 7 days after T-cell infusion and continued through the latest time-point (30 days).

Regarding antigen-specific responses, SMART-DCs-pp65 administration enabled pp65-specific antigen presentation simultaneously through HLA-B*0702 and HLA-A*0201 MHC class I molecules. A relatively dominant T-cell response to pp65 through HLA-B*07 over HLA- A*0201 has been documented (Lacey et al., 2003), which might indicate that SMART-DCs have a versatile antigen-presentation capability through different MHC class I molecules.

To our knowledge, this is the first demonstration that antigen-specific human $\mathrm{CD}^{+}{ }^{+} \mathrm{T}$ cells stimulated in vivo could be directly detected in the peripheral blood, thus bypassing the need for additional in vitro restimulation. In addition, splenocytes collected 14 days after T-cell infusion and in vitro

FIG. 8. T-cell expansion and activation of pp65-specific $\mathrm{CD}^{+} \mathrm{T}$-cell responses in mice receiving different treatments: Monocytes expressing LV-pp65 (Mono LV-pp65), cDCs loaded with pp65 peptides (cDC pp65pp), cDCs expressing LV-pp65 (cDC LV-pp65), SMART-DCs, and SMART-DCspp65. Frequency of human $\mathrm{CD}^{+} / \mathrm{CD}^{+} \mathrm{T}$ cells (a) and human $\mathrm{CD}^{+} / \mathrm{CD}^{+} \mathrm{T}$ cells $(\mathbf{b})$ in peripheral blood obtained from mice ( $n=3$ per group) that were administered with APCs 7 days prior to T-cell infusion (left panel) or injected with APCs and T cells concurrently (right panel) and. Flow cytometry analyses were performed 10 days after T-cell infusion. (c) IFN $\gamma$-ELISPOT assay was performed with human $\mathrm{CD}^{+}$cells isolated from spleen 10 days after T-cell infusion. Averages of detectable spot counts (per $10^{4}$ seeded cells) are shown. (d) Frequencies of $\mathrm{T}$ cells analyzed from peripheral blood and reactive against $A^{*} 0201$ restricted NLVPMVATV (diagonal-patterned bars) and $\mathrm{B}^{* 0702}$ restricted TPRVTGGGAM (black) tetramers are shown. Percentages represent frequencies of tetramer positive $/ \mathrm{CD}^{+} /$ $\mathrm{CD}^{+} \mathrm{T}$ cells. Error bars indicate standard deviation (SD) $n=3$ mice pre group ${ }^{*} p<0.05$. 
stimulated (overnight) with pp65 epitopes demonstrated the IFN $-\gamma$ production through ELISPOT assay, confirming the functionality of the in vivo expanded effector CTLs. Associated with the anti-pp65 antigenic responses, we observed a significant increase in the frequency of effector memory $\mathrm{T}$ cells in the spleen of mice immunized with SMART-DCs-pp65.

With the results obtained, a tentative model representing the different outcomes of $\mathrm{T}$ cells infused in vivo can be proposed. It is possible that, in the absence of human cognate APCs, T cells infused intravenously will mostly localize to the spleen and liver simply due to a "first-pass" effect (see schematic representation, Supplementary Fig. S7). Once the human $\mathrm{T}$ cells become trapped in these organs they will be highly stimulated against xenogenic mouse antigens and, apparently, the $\mathrm{CD} 4^{+} \mathrm{T}$-cell population is favored in this process. In contrast, SMART-DCs injected subcutaneously determined a homeostatic expansion: $\mathrm{T}$ cells that were initially circulating or were trapped in spleen were recruited to the DC implantation sites, where they continued to be costimulated through human cytokines and MHC molecules (Supplementary Fig. S7). Lastly, SMART-DCs-pp65 presenting antigen that could be clearly recognized by autologous $\mathrm{CD}^{+} \mathrm{T}$ memory cells generated a stronger recruitment of lymphocytes to the injection sites and subsequently a systemic redistribution of cells in the spleen and in the bloodstream (Supplementary Fig. S7). Short-lived cDCs (loaded with pp65 epitopes or transduced for pp65 expression) and administered prior to or concurrently with $\mathrm{T}$ cells showed much lower capacity to recruit $\mathrm{T}$ cells and stimulated their expansion, biodistribution, and antigen-specific stimulation compared with SMART-DCs-pp65. Interestingly, administration of SMART-DCs-pp65 at the same time as T cells produced inferior results, which indicates that allowing self-differentiation of the DCs enables stronger effects towards T cells.

These data ultimately support our proposed approach for in vivo preconditioning of the host with long-lived and stable DCs prior to T-cell infusion, such that the T cells can find optimal conditions in the host for both homeostatic expansion and antigenic engagements. As a general consequence, as predicted by forefront observers in the field, long-lived autologous human APCs represented here by SMART-DCs expressing human cytokines and MHC molecules enabled beneficial homeostatic conditions for human T-cell engraftment and maintenance in immunodeficient mice.

Importantly, within the duration of the experiments outlined here (up to 30 days), we observed very sparse (less than $20 \%$ ) occurrence of unexplained death, which could be in part attributed to side effects due to prolonged general anesthesia required for the imaging analyses. Clinical signs of GvHD were not observed.

Beyond the novel experimental system presented and discussed, the results obtained could also imply future clinical developments of SMART-DCs as a prophylactic cell product against cancer and/or chronic infections after hematopoietic stem cell transplantation. The need for high clinical-grade numbers of ex vivo-expanded CTL for adoptive transfer implies prolonged culture periods and delays its clinical benefit, resulting in difficulty translating this therapeutic option to routine clinical practice.

Despite the promising results shown here in this shortterm PBMC infusion xenogenic model, clinical translation of
SMART-DCs for homeostatic lymphocytic regeneration after transplantation is still premature, due to the fear of overt T-cell expansion causing GvHD. Therefore, future nonclinical studies using fully humanized mice (transplanted with human hematopoietic stem cells) will be warranted for the evaluation of the long-term effects of SMART-DC administration in the hematopoietic stem cell transplantation setting.

SMART-DCs are post-mitotic cells with a low risk of malignancy development, and GM-CSF and IL-4 have been used systemically as drugs with no major toxicity effects. Although LVs have not been tested clinically as an immunotherapeutic cell product, current efforts for improving LV safety (such as integration-deficient LVs) and for establishing clinical grade LV production are ongoing (Pincha et al., 2010). Thus, standardized production, conforming to good manufacturing practice, of lentivirus-transduced DCs for their use in cancer and infectious disease immunotherapy is an ongoing effort in several laboratories, including ours.

\section{Acknowledgments}

G.S. designed and performed experiments, analyzed data, prepared the figures and wrote the first draft; R.S. designed experiments, analyzed data, completed and revised the manuscript. We thank all members of the Lymphatic Cell Therapy laboratory for their contributions to the completion of this work. We want to specially thank Dr. Constança Figueiredo from the Institute for Transfusion Medicine for performing the Luminex bead arrays. We also thank the MHH sorting facility, the Institute for Virology (Dr. Benno Wölk) and Transfusion Medicine for their professional support. We thank Prof. Oliver Pabst for helpful assistance with the histological analyses. The authors gratefully acknowledge Prof. Dr. Maria Villacres (University of Southern California), Prof. Dr. Philipp Beckhove (University of Heidelberg), Prof. Dr. Michael Roth (University of California, Los Angeles), Prof. Dr. Sylvia Kiertscher (University of California, Los Angeles) and Dr. Airi Harui (University of California, Los Angeles) for critical reading of the manuscript. This work was supported by grants of the German Research Council (DFG/ SFB738 to R.S.), a collaborative research grant from the Jose Carreras Foundation (to R.S.) and a research grant from the Deutsche Krebshilfe (to R. S.). R.S. is supported by a DFG/ REBIRTH Professorship in Regenerative Medicine and S.B. is recipient of a postdoctoral fellowship from BMBF/ IFB-TX.

\section{Author Disclosure Statement}

The authors declare that no competing financial interests exist.

\section{References}

Anthony, D.D., Yonkers, N.L., Post, A.B., et al. (2004). Selective impairments in dendritic cell-associated function distinguish hepatitis C virus and HIV infection. J. Immunol. 172, 49074916.

Borst, E.M., Benkartek, C., and Messerle, M. (2007). Use of bacterial artificial chromosomes in generating targeted mutations in human and mouse cytomegaloviruses. Curr. Protoc. Immunol. Chapter 10, Unit 1032.

De Vries, I.J., Krooshoop, D.J., Scharenborg, N.M., et al. (2003). Effective migration of antigen-pulsed dendritic cells to lymph 
nodes in melanoma patients is determined by their maturation state. Cancer Res. 63, 12-17.

Figdor, C.G., De Vries, I.J., Lesterhuis, W.J., and Melief, C.J. (2004). Dendritic cell immunotherapy: mapping the way. Nat. Med. 10, 475-480.

Gorantla, S., Santos, K., Meyer, V., et al. (2005). Human dendritic cells transduced with herpes simplex virus amplicons encoding human immunodeficiency virus type 1 (HIV-1) gp120 elicit adaptive immune responses from human cells engrafted into NOD/SCID mice and confer partial protection against HIV-1 challenge. J. Virol. 79, 2124-2132.

Harui, A., Kiertscher, S.M., and Roth, M.D. (2011). Reconstitution of huPBL-Nsg mice with donor-matched dendritic cells enables antigen-specific T-cell activation. J. Neuroimmune Pharmacol. 6, 148-157.

Horvath, R., Budinsky, V., Kayserova, J., et al. (2009). Kinetics of dendritic cells reconstitution and costimulatory molecules expression after myeloablative allogeneic haematopoetic stem cell transplantation: implications for the development of acute graft-versus host disease. Clin. Immunol. 131, 60-69.

Inoue, M., Senju, S., Hirata, S., et al. (2009). An in vivo model of priming of antigen-specific human CTL by Mo-DC in NOD/ Shi-scid IL2rgamma(null) (NOG) mice. Immunol. Lett. 126, 67-72.

Kimura, T., Koya, R.C., Anselmi, L., et al. (2007). Lentiviral vectors with CMV or MHCII promoters administered in vivo: immune reactivity versus persistence of expression. Mol. Ther. $15,1390-1399$.

Kolb, H.J. (2008). Graft-versus-leukemia effects of transplantation and donor lymphocytes. Blood 112, 4371-4383.

Korin, Y.D., and Zack, J.A. (1998). Progression to the G1b phase of the cell cycle is required for completion of human immunodeficiency virus type 1 reverse transcription in T cells. J. Virol. 72, 3161-3168.

Koya, R.C., Weber, J.S., Kasahara, N., et al. (2004). Making dendritic cells from the inside out: lentiviral vector-mediated gene delivery of granulocyte-macrophage colony-stimulating factor and interleukin 4 into CD14 + monocytes generates dendritic cells in vitro. Hum. Gene Ther. 15, 733-748.

Koya, R.C., Kimura, T., Ribas, A., et al. (2007). Lentiviral vectormediated autonomous differentiation of mouse bone marrow cells into immunologically potent dendritic cell vaccines. Mol. Ther. 15, 971-980.

Lacey, S.F., Villacres, M.C., La Rosa, C., et al. (2003). Relative dominance of HLA-B*07 restricted CD8 + T-lymphocyte immune responses to human cytomegalovirus pp65 in persons sharing HLA-A*02 and HLA-B*07 alleles. Hum. Immunol. 64, 440-452.

Lapenta, C., Santini, S.M., Spada, M., et al. (2006). IFN-alphaconditioned dendritic cells are highly efficient in inducing cross-priming CD8(+) T cells against exogenous viral antigens. Eur. J. Immunol. 36, 2046-2060.

Maurice, M., Verhoeyen, E., Salmon, P., et al. (2002). Efficient gene transfer into human primary blood lymphocytes by surface-engineered lentiviral vectors that display a $\mathrm{T}$ cellactivating polypeptide. Blood 99, 2342-2350.

Merad, M., and Manz, M.G. (2009). Dendritic cell homeostasis. Blood 113, 3418-3427.

Pearson, T., Shultz, L.D., Miller, D., et al. (2008). Non-obese diabetic-recombination activating gene-1 (NOD-Rag1 null) interleukin (IL)-2 receptor common gamma chain (IL2r gamma null) null mice: a radioresistant model for human lymphohaematopoietic engraftment. Clin. Exp. Immunol. 154, 270-284.

Pincha, M., Sundarasetty, B.S., and Stripecke, R. (2010). Lentiviral vectors for immunization: an inflammatory field. Expert Rev. Vaccines 9, 309-321.

Pincha, M., Salguero, G., Wedekind, D., et al. (2011). Lentiviral vectors for induction of self-differentiation and conditional ablation of dendritic cells. Gene Ther. Mar 17. [Epub ahead of print] PubMed PMID: 21412283.

Small, T.N., Papadopoulos, E.B., Boulad, F., et al. (1999). Comparison of immune reconstitution after unrelated and related T-cell-depleted bone marrow transplantation: effect of patient age and donor leukocyte infusions. Blood 93, 467-480.

Stripecke, R. (2009). Lentiviral vector-mediated genetic programming of mouse and human dendritic cells. Methods Mol. Biol. 506, 139-158.

Sylwester, A.W., Mitchell, B.L., Edgar, J.B., et al. (2005). Broadly targeted human cytomegalovirus-specific CD4+ and CD8 + T cells dominate the memory compartments of exposed subjects. J. Exp. Med. 202, 673-685.

Szymczak, A.L., and Vignali, D.A. (2005). Development of 2A peptide-based strategies in the design of multicistronic vectors. Expert Opin. Biol. Ther. 5, 627-638.

Szymczak, A.L., Workman, C.J., Wang, Y., et al. (2004). Correction of multi-gene deficiency in vivo using a single 'selfcleaving' 2A peptide-based retroviral vector. Nat. Biotechnol. 22, 589-594.

Williams, K.M., Hakim, F.T., and Gress, R.E. (2007). T cell immune reconstitution following lymphodepletion. Semin. Immunol. 19, 318-330.

Yamashita, K., Choi, U., Woltz, P.C., et al. (2004). Severe chronic graft-versus-host disease is characterized by a preponderance of $\mathrm{CD} 4(+)$ effector memory cells relative to central memory cells. Blood 103, 3986-3988.

Yoshida, A., Tanaka, R., Murakami, T., et al. (2003). Induction of protective immune responses against R5 human immunodeficiency virus type 1 (HIV-1) infection in hu-PBL-Scid mice by intrasplenic immunization with HIV-1-pulsed dendritic cells: possible involvement of a novel factor of human CD4(+) Tcell origin. J. Virol. 77, 8719-8728.

Address correspondence to: Dr. Renata Stripecke Laboratory of Lymphatic Cell Therapy Department of Hematology, Hemostasis, Oncology and Stem Cell Transplantation Medical School Hannover Hans Borst Zentrum, Carl Neuberg Strasse 1 Building J11, Floor 02, Room 6090/6099 D-30625 Hannover Germany

E-mail: stripecke.renata@mh-hannover.de

Received for publication November 8, 2010; accepted after revision May 16, 2011.

Published online: May 16, 2011. 